\title{
Development of dextrin-amphotericin B formulations for the treatment of Leishmaniasis
}

\author{
R. Silva-Carvalho a , J. Fidalgo ${ }^{\text {a }}$, K.R. Melo ${ }^{\text {b }}$, M.F. Queiroz ${ }^{\text {b }}$, S. Leal ${ }^{a}$, H.A. Rocha ${ }^{\text {b }}$, T. Cruz ${ }^{\text {c,d }}$, P. Parpot ${ }^{\text {a,e }}$, \\ A.M. Tomás ${ }^{\mathrm{c}, \mathrm{d}, \mathrm{f}}$, M. Gama ${ }^{\mathrm{a}, *}$ \\ a CEB - Centre of Biological Engineering, University of Minho, Campus de Gualtar, 4710-057 Braga, Portugal \\ b Departamento de Bioquímica, Centro de Biociências, Universidade Federal do Rio Grande do Norte, Natal, RN, Brazil \\ c i3S - Instituto de Investigação e Inovação em Saúde, Universidade do Porto, Rua Alfredo Allen 208, 4200-135 Porto, Portugal \\ d IBMC - Instituto de Biologia Molecular e Celular, Universidade do Porto, Rua Alfredo Allen 208, 4200-135 Porto, Portugal \\ e Centre of Chemistry, University of Minho, Campus de Gualtar, 4710-057 Braga, Portugal \\ f ICBAS - Instituto de Ciências Biomédicas Abel Salazar, Universidade do Porto, Rua de Jorge Viterbo Ferreira 228, 4050-313 Porto, Portugal
}

\section{A R T I C L E I N F O}

\section{Article history:}

Received 17 December 2019

Received in revised form 1 March 2020

Accepted 3 March 2020

Available online 05 March 2020

\section{Keywords:}

Dextrin

Amphotericin B

Leishmaniasis

\begin{abstract}
A B S T R A C T
The most effective medicines available for the treatment of leishmaniasis, a life-threatening disease, exhibit serious toxicological issues. To achieve better therapeutic efficiency while decreasing toxicity associated with amphotericin B (AmB), water-soluble dextrin-AmB (Dex-AmB) formulations were developed. Self-assembled nanocomplexes were formed by dissolving Dex and $A m B$ in alkaline borate buffer, followed by dialysis and either freeze-drying (FD) or nano spray-drying (SD), yielding water dispersible particles with a diameter of $214 \mathrm{~nm}$ and $347 \mathrm{~nm}$, respectively. The very simple production process allowed the formation of amorphous inclusion complexes containing $14 \%$ of $\mathrm{AmB}$ in the form of monomers and water-soluble aggregates. Nanocomplexes were effective against parasites in axenic culture ( $\mathrm{IC}_{50}$ of 0.056 and $0.096 \mu \mathrm{M}$ for $L$. amazonensis and 0.030 and $0.044 \mu \mathrm{M}$ for $L$. infantum, respectively for Dex-AmB FD and Dex-AmB SD) and in decreasing the intramacrophagic infection with $L$. infantum ( $\mathrm{IC}_{50}$ of 0.017 and $0.023 \mu \mathrm{M}$, respectively for Dex-AmB FD and Dex-AmB SD). Also, the formulations were able to significantly reduce the cytotoxicity of AmB. Overall, this study demonstrates the suitability of dextrin as an AmB carrier and the facile and inexpensive development of a delivery system for the treatment of leishmaniasis.
\end{abstract}

(c) 2020 Elsevier B.V. All rights reserved.

\section{Background}

Leishmaniasis has been classified as one of the most neglected tropical morbidity and mortality causing diseases [1]. In fact, about 12-15 million people are currently infected and 350 million people in 89 countries mainly in tropical and subtropical areas live at risk of developing one of the many forms of the disease [1-3]. Protozoan parasites of the genus Leishmania, which are responsible for this life-threatening disease [4], can maintain their life cycle through transmission between two forms: the extracellular motile promastigote that multiplies in the gut of Phlebotomus and/or Lutzomyia sandflies [4,5], and the intracellular non-motile amastigote which replicates inside macrophages of mammalian hosts [6]. These parasites can cause a plethora of pathological manifestations such as cutaneous, mucocutaneous or diffuse cutaneous leishmaniasis, characterized by localized symptoms in the skin or mucosal surfaces. Other Leishmania species that are more aggressive, target internal organs causing visceral leishmaniasis (associated to approximately 70,000 deaths per year) $[2,6]$.

Despite the well-established knowledge and the recent advance in our understanding of leishmaniasis biology, some aspects of this disease remain enigmatic and the current control or treatment strategies are rather inadequate [6,7]. This is due both to limited availability of

Abbreviations: AmB, Amphotericin B; BMMФ, Bone marrow-derived macrophages; $\mathrm{CC}_{50}$, cytotoxicity concentration 50\%; Cryo-SEM, Scanning Electron Cryomicroscopy; DAPI, 4',6-

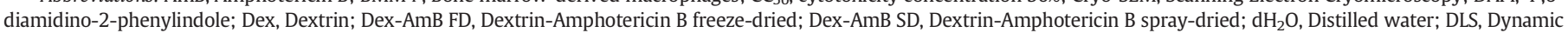

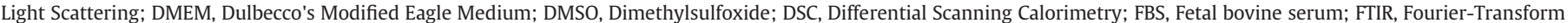

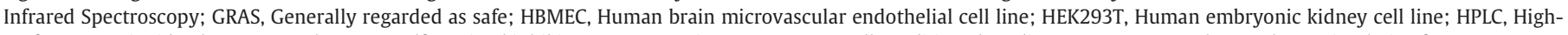

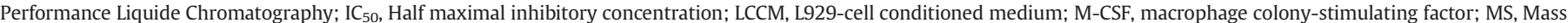

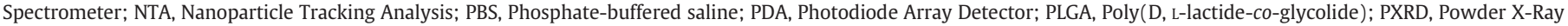
Diffraction; RBCs, Red blood cells; RPMI, Roswell Park Memorial Institute 1640; SEM, Scanning Electron Microscope; SI, Selectivity index; UV, Ultraviolet.

* Corresponding author at: Department of Biological Engineering, University of Minho, Campus de Gualtar, 4710-057 Braga, Portugal.

E-mail address: fmgama@deb.uminho.pt (M. Gama). 
effective parenteral drug formulations and constant appearance of resistant parasites. Besides, as there are currently no effective vaccines to prevent human leishmaniasis, the cure relies on chemotherapy, using drugs of high toxicity, low efficacy and difficult to administer due to low solubility $[2,8]$. Amphotericin B (AmB) interacts with cell membrane sterols promoting cell death [9-11]. This drug is recommended as a second-line treatment for visceral and mucocutaneous leishmaniasis, but it presents toxicity and side effects mainly related to the kidneys, central nervous system and liver [8]. Strategies to overcome these shortcomings, such as combination therapy, modification of AmB or drug delivery systems have been the cornerstones to improve the therapeutic efficacy and reduce the toxicity. Some marketed AmB-based products are available (e.g. Fungizone ${ }^{\circledR}$ and Ambisome ${ }^{\circledR}$ ) which, despite higher efficiency, still raise concerns regarding toxicity and high production costs $[8,12]$. Therefore, it is of urgent need the development of alternative effective strategies to facilitate use of AmB for leishmaniasis treatment.

Different polymers have been widely used as drug carriers [13]. In fact, polysaccharides (e.g. arabinogalactan $[8,14,15]$, dextran $[16,17]$, gum arabic [18], sodium alginate [19], pectin [20], galactomannan [21]) and synthetic polymers (e.g. polymannose [22] and polyglucose [23]) bearing high water-solubility, low toxicity, a high degree of biodegradability and biocompatibility [15], have attracted considerable attention in recent years for producing water-soluble polymer-drug formulations. Besides drug targeting, the use polysaccharides enhances drug solubility and stability, allowing prolonged circulation lifetime and activity and reduction of drug toxicity [8]. Nevertheless, most of the polysaccharide-based AmB formulations reported in literature are prepared by reductive amination where polysaccharides are oxidized to render di-aldehyde derivatives reactive with $A m B$ via Schiff base (imine bond) or via amine bond (reduction) [14]. In this work, we hypothesize that dextrin, a class of low molecular weight carbohydrates, may be used for development of an AmB drug delivery system as it is biocompatible, non-immunogenic and degradable in vivo by amylases [24]. Further, we demonstrate that time consuming and complex steps involving a pre-oxidation of the polysaccharides are not required to obtain a polysaccharide-AmB formulation. We show that by simply mixing and processing new water-soluble dextrin-amphotericin B (Dex and $\mathrm{AmB}$ ) in a freeze-drier or spray-dyer, Dex-AmB nanocomplexes with a promising role in the treatment of leishmaniasis can be produced.

\section{Material and methods}

\subsection{Reagents}

Amphotericin B (AmB, molecular weight of $924.08 \mathrm{~g} / \mathrm{mol}$ ) powder from Streptomyces sp., resazurin Sodium salt, 4',6-diamidino-2phenylindole (DAPI), triton X-100, paraformaldehyde, Schneider's Insect medium, sodium tetraborate decahydrate, potassium bromide, phenol red and hemin were purchased from Sigma-Aldrich (Missouri, USA). Dextrin Tackidex ${ }^{\circledR}$ (medical grade dextrin from potato starch) with a degree of polymerization (DP) of 16 glucose residues and a branching degree of $9 \%$ was a gift from Roquette (Lestrem, France). Dulbecco's Modified Eagle Medium (DMEM), fetal bovine serum (FBS) and penicillin-streptomycin were obtained from Merck Millipore (Massachusetts, USA). Roswell Park Memorial Institute (RPMI) 1640 Glutamax supplemented medium and L-glutamine (GlutaMAX ${ }^{\mathrm{TM}}-\mathrm{I}$ ) was purchased from Gibco (Massachusetts, USA). Dimethylsulfoxide (DMSO ATCC $® 4-\mathrm{X}^{\mathrm{TM}}$ ) solution for cell culture was acquired from American Type Culture Collection (ATCC, Virginia, USA). Dialysis tubing with a molecular weight cut-off of 1000 Da was obtained from Orange Scientific (Braine-l'Alleud, Belgium). High-content screening (HCS) CellMask ${ }^{\mathrm{TM}}$ Deep Red stain was acquired from Invitrogen (California, USA). Acetonitrile and Formic acid were purchased from Fisher Scientific (New Hampshire, USA).

\subsection{Dextrin-amphotericin B production}

Before the procedure, $1.5 \mathrm{~g}$ of dextrin (apparent molecular weight of $4.5 \mathrm{kDa}$ [24]) was diluted in distilled water $\left(\mathrm{d}_{2} \mathrm{O}\right)$ at a concentration of $10 \mathrm{mg} / \mathrm{mL}$, dialyzed using a dialysis membrane with a molecular weight cut-off $1000 \mathrm{Da}$ against $\mathrm{dH}_{2} \mathrm{O}$ for $72 \mathrm{~h}$ and then freeze-dried. Freezedried dextrin (Dex) was mixed with amphotericin $B(A m B)$ in a mass ratio of $4: 1$ and dissolved in $32 \mathrm{~mL}$ of $0.1 \mathrm{M}$ borate buffer ( $\mathrm{pH} 11$ ), resulting in a final concentration of $2.5 \mathrm{mg} / \mathrm{mL}$ of $\mathrm{AmB}$ in the solution. The mixture, protected from light, was gently stirred at $4{ }^{\circ} \mathrm{C}$ for $48 \mathrm{~h}$ and then dialyzed at $4{ }^{\circ} \mathrm{C}$ against $\mathrm{dH}_{2} \mathrm{O}$ using a membrane with a molecular weight cut-off $1000 \mathrm{Da}$. During this process, the $\mathrm{pH}$ was kept at \pm 6 . Afterward, the solution was freeze-dried (Coolsafe 100-9 Pro, Labogene, Allerød, Denmark) to obtain a yellow-orange material (Dex-AmB FD) or processed in a Nano Spray Dryer B-90 HP (Büchi Labortechnik AG, Flawil, Switzerland), yielding a yellow-orange powder (Dex-AmB SD). Briefly, in the latter case the solution was fed into the instrument by a peristaltic pump and sprayed using a piezoelectric nebulizer, operated at a vibrational frequency of $120 \mathrm{kHz}$, with mean micron-sized pores of $2-25 \mu \mathrm{m}$. The feeding rate was set to $40 \%$, where the gas flow rate was adjusted at $10 \mathrm{~L} / \mathrm{min}$. The inlet temperature was $120^{\circ} \mathrm{C}$. The obtained powder was separated, collected from the drying chamber and stored at $4{ }^{\circ} \mathrm{C}$ in a recipient containing silica gel. Dex without AmB was also processed by freeze-drying (Dex FD) or nano spray-drying (Dex SD), to be tested as control.

\subsection{Dex-AmB characterization}

\subsubsection{Nanoparticle tracking analysis (NTA)}

Size of the nanocomplexes was measured by the laser-scattering technique Nanoparticle Tracking Analysis (NTA) using a NanoSight NS 500 (Malvern Instruments Ltd., Worcestershire, UK) equipped with a sample chamber and a $640 \mathrm{~nm}$ laser. The produced material was dissolved at $1 \mathrm{mg} / \mathrm{mL}$ in $\mathrm{dH}_{2} \mathrm{O}$. The solutions $(0.3 \mathrm{~mL})$ were introduced in the sample chamber and analyzed for $60 \mathrm{~s}$, using manual shutter and gain adjustments. Measurements were performed in triplicate at room temperature and the NTA 2.3 Build 0017 software (Malvern) was used to collect the data.

\subsubsection{Zeta potential}

The zeta potential of Dex-AmB nanocomplexes was estimated by Dynamic Light Scattering (DLS) using a Zetasizer NanoZS, (Malvern Instruments Ltd., Worcestershire, UK). Three measurements of each solution were performed and the zeta potential values were determined using Henry's equation [25].

\subsubsection{Morphology analysis}

2.3.3.1. Scanning electron cryomicroscopy (Cryo-SEM). Dex-AmB nanocomplexes were dispersed in $\mathrm{dH}_{2} \mathrm{O}$ at a concentration of $2 \mathrm{mg} / \mathrm{mL}$ and rapidly cooled plunging it into sub-cooled nitrogen. The samples were transferred under vacuum to the cryo stage (Gatan, Alto 2500, UK) and then fractured and sublimated for $120 \mathrm{~s}$ at $-90{ }^{\circ} \mathrm{C}$ to remove the superficial ice layer and allow the exposure of the nanocomplexes. Then, the samples were sputter-coated with gold and palladium for $45 \mathrm{~s}$ and transferred to the observation chamber of an electron microscope (SEM/EDS: JEOL JSM 6301F/Oxford Inca Energy 350 from the Laboratory for Scanning Electron Microscopy and X-ray Microanalysis at Materials Centre of the University of Porto (CEMUP)). The observation was performed at $-150{ }^{\circ} \mathrm{C}$ and $15 \mathrm{kV}$.

2.3.3.2. Scanning electron microscopy (SEM). The powder material produced in the nano spray dryer was observed using a scanning electron microscopy (SEM; FEI Quanta 650 FEG Environmental, Field Electron and Ion Company, Oregon, USA). Samples were fixed using a doublesided electrically conductive carbon adhesive tape (PELCO Tabs ${ }^{\mathrm{TM}}$, 
California, USA) on an aluminum pin stub and then sputter coated with a 20 Å gold layer. Images were obtained at an excitation voltage of $5 \mathrm{kV}$.

\subsubsection{HPLC-PDA-MS analysis}

The HPLC system Finnigan LXQ from ThermoElectron Corporation (Thermo Fisher Scientific, Massachusetts, USA) equipped with a Surveyor Autosampler (Thermo fisher Scientific, Massachusetts, USA) was used to quantify the AmB content in the produced materials. The analytical column used was a Kinetex® C18 $100 \AA 100 \times 4.6 \mathrm{~mm}(2.6 \mu \mathrm{m}$ particle size) from Phenomenex (California, USA) and the analysis was performed using an ion trap Mass Spectrometer (MS) equipped with an ElectroSpray Ionization (ESI) source (Thermo Fisher Scientific, Massachusetts, USA) and a 2998 photodiode array (PDA) wavelength detector (Waters Corporation, Massachusetts, USA). The MS analysis was operated using Selected Ion Monitoring (SIM) Mode with scan ranges from 923 to $925 \mathrm{~m} / z$, and the instrument was operated in positive ionization mode. The UV detection wavelengths were defined to 387 and $408 \mathrm{~nm}$. The mobile phase was composed by two filtered and degassed solvents: (A) ultrapure water with $0.1 \%(\mathrm{v} / \mathrm{v})$ formic acid and (B) acetonitrile with $0.1 \%(\mathrm{v} / \mathrm{v})$ formic acid. The solvent gradient started with $80 \% \mathrm{~A}$ and $20 \% \mathrm{~B}$, reaching $10 \% \mathrm{~A}$ and $90 \% \mathrm{~B}$ at $15 \mathrm{~min}$ and $80 \% \mathrm{~A}$ and $20 \% \mathrm{~B}$ at $25 \mathrm{~min}$, condition that was maintained until the end of the analysis. The run time was $30 \mathrm{~min}$, with a flow rate of $0.3 \mathrm{~mL} / \mathrm{min}$ and the analysis was performed at a $25{ }^{\circ} \mathrm{C}$ with a sample injection volume of $25 \mu \mathrm{L}$. Moreover, the source dependent parameters such as Sheath Gas flow rate, Auxiliar Gas flow rate, Sweep Gas flow rate, Tube Lens, Capillar Voltage, Capillar Temperature and Spray Voltage were optimized to $50,10,10,115 \mathrm{~V}, 29 \mathrm{~V}, 250{ }^{\circ} \mathrm{C}$ and $5000 \mathrm{~V}$, respectively. All spectral data was acquired and analyzed using Xcalibur Chromatography Software (Thermo Fisher Scientific, Massachusetts, USA).

The stock standard solution of AmB $(150 \mu \mathrm{g} / \mathrm{mL})$ and Dex-AmB nanocomplexes ( $3 \mathrm{mg} / \mathrm{mL}$ ) were prepared by dissolving 6 and $9 \mathrm{mg}$ of each material in 40 and $3 \mathrm{~mL}$ of acetonitrile:ultrapure water:formic acid (39.22:58.33:2.45\% (v/v)) respectively, under mild stirring and protected from light during $2 \mathrm{~h}$ at room temperature. Subsequently, the calibration curves were obtained using seven standard solutions for the MS analysis - 2, 5, 10, 20 60, 80 and $120 \mu \mathrm{g} / \mathrm{mL}$ - and five standard solutions for the UV analysis - $0.5,1,2,10$ and $20 \mu \mathrm{g} / \mathrm{mL}$ - and were prepared from the stock standard solution using acetronitrile:ultrapure water (39.22:60.78\% (v/v)). Similarly, for the Dex-AmB nanocomplexes, solutions of 25,50 and $100 \mu \mathrm{g} / \mathrm{mL}$ were prepared using the abovementioned solvent. Prior to injection, standards and samples were filtered through a $0.22 \mu \mathrm{m}$ pore size Nylon filter (Tecnocroma, Caldas da Rainha, Portugal). A linear regression was used to determine slopes, intercepts and correlation coefficients. The percentage of drug association efficiency ( $\mathrm{AE} \%)$ as well as the drug content $(\% \mathrm{w} / \mathrm{w})$ in the produced nanocomplexes were determined using the following equations, respectively:

$A E(\%)=\frac{m_{A m B}}{m_{\text {AmB_initial }}} \times 100$

$A m B$ Content $(\%)=\frac{m_{A m B}}{m_{D e x-A m B}} \times 100$

where $\mathrm{m}_{\mathrm{AmB} \_ \text {initial }}$ is the initial mass of $\mathrm{AmB}$ used in the process, $m_{A m B}$ is the AmB mass in the final formulation that was obtained via HPLC-MS and HPLC-PDA analysis and $m_{D e x-A m B}$ is the final mass of the produced material.

\subsubsection{Fourier-transform infrared spectroscopy (FTIR)}

FTIR spectroscopic studies of free-AmB, Dex FD, Dex SD, Dex-AmB FD and Dex-AmB SD were carried out employing an ABB Bomem FTLA2000-104 spectrophotometer (Québec, Canada). About $2 \mathrm{mg}$ of the different samples was mixed with $200 \mathrm{mg}$ of dry potassium bromide to obtain a solid pellet. Samples were examined at transmission mode and 64 scans were co-added for each spectrum over a wavenumber range of $4000-700 \mathrm{~cm}^{-1}$.

\subsubsection{Differential scanning calorimetry (DSC)}

DSC thermograms of AmB, Dex FD, Dex SD, Dex-AmB FD and DexAmB SD were obtained by using a DSC 600 Perkin-Elmer (Massachusetts, USA). An empty aluminum pan was used to calibrate the apparatus. A known amount of samples equivalent to $0.7 \mathrm{mg}$ of $\mathrm{AmB}$ were weighed and hermetically sealed in standard aluminum pans. Samples were scanned from 25 to $235^{\circ} \mathrm{C}$ at a ramp speed of $10{ }^{\circ} \mathrm{C} / \mathrm{min}$ under nitrogen purge with a flow rate of $20 \mathrm{~mL} / \mathrm{min}$. All samples were analyzed in triplicate.

\subsubsection{Powder X-ray diffraction (PXRD)}

PXRD patterns of the dried samples (AmB, Dex FD, Dex SD, Dex-AmB FD and Dex-AmB SD) were recorded using a PANalytical X'Pert Pro Xray diffractometer equipped with $X^{\prime}$ Celerator detector and secondary monochromator (Malvern Panalytical, United Kingdom). For the analysis, the samples were placed in a Bragg-Bentano geometry and scanned from $7^{\circ}$ to $50^{\circ} 2 \theta$ angular range using a $\mathrm{Cu} \mathrm{K} \alpha$ radiation ( $40 \mathrm{kV}$ operating voltage; $30 \mathrm{~mA}$ current).

\subsubsection{Spectrophotometric analysis of Dex-AmB nanocomplexes}

$\mathrm{AmB}$ and Dex-AmB nanocomplexes were analyzed by UV-vis spectroscopy in the range of 300-450 nm using a UV-visible spectrophotometer (JASCO V-560 series, Jasco Inc., Maryland, USA) operated at a band width of $5 \mathrm{~nm}$, data pitch of $1 \mathrm{~nm}$ and scanning speed of $400 \mathrm{~nm} / \mathrm{min}$. The nanocomplexes were dissolved in $\mathrm{dH}_{2} \mathrm{O}$ at the final AmB concentration of $144 \mu \mathrm{M}\left(1 \mathrm{mg}_{\text {nanocomplex }} / \mathrm{mL}\right)$ and then, to avoid spectrum saturation, samples were diluted to $10 \mu \mathrm{M}$ using $\mathrm{dH}_{2} \mathrm{O}$. A stock solution of AmB at $1082 \mu \mathrm{M}(1 \mathrm{mg} / \mathrm{mL})$ was prepared using $0.1 \mathrm{M}$ borate buffer $\mathrm{pH} 11$. Then, as performed for the nanocomplexes, this solution was diluted to $10 \mu \mathrm{M}$ using $\mathrm{dH}_{2} \mathrm{O}$. A volume of $200 \mu \mathrm{L}$ of the solutions was used for the measurements in a quartz cuvette. The ratio of the absorbance of peak I (315-350) to the peak IV (408-410) was used to monitor the aggregation state of AmB.

\subsection{In vitro assays}

\subsubsection{Culture of Leishmania parasites}

Leishmania infantum promastigotes (MHOM/MA/67/ITMAP-263) were cultured in RPMI 1640 Glutamax supplemented with $10 \%(\mathrm{v} / \mathrm{v})$ iFBS, $50 \mathrm{U} / \mathrm{mL}$ penicillin, $50 \mu \mathrm{g} / \mathrm{mL}$ streptomycin and $20 \mathrm{mM}$ HEPES pH 7.4 (complete RPMI medium). Leishmania amazonensis promastigotes (MHOM/BR/LTB0016) were cultured in Schneider's Insect medium supplemented with $10 \%(\mathrm{v} / \mathrm{v})$ heat inactivated FBS (iFBS), $100 \mathrm{U} / \mathrm{mL}$ penicillin, $100 \mu \mathrm{g} / \mathrm{mL}$ streptomycin, $5 \mathrm{mM}$ HEPES $\mathrm{pH} 7.4$ and $5 \mu \mathrm{g} / \mathrm{mL}$ phenol red (complete Schneider's insect medium). Both parasite strains were cultured at $25^{\circ} \mathrm{C}$. Infectivity was secured as previously described by Gomes-Alves et al. [26].

Axenic amastigotes of $L$. infantum were obtained as described in the literature [27]. Axenic L. infantum amastigotes were derived from the above referred strain by maintaining the culture at $37{ }^{\circ} \mathrm{C}$ with $5 \% \mathrm{CO}_{2}$ in MAA/20 medium (medium for axenically grown amastigotes) supplemented with $20 \%$ (v/v) iFBS, 2 mM L-Glutamine and $0.023 \mathrm{mM}$ hemin. Purity, assessed by microscopy on the basis of morphology (round and immobile organisms, about 2-3 $\mu \mathrm{m}$ and without visible flagella), was around $95 \%$.

\subsubsection{Effect of Dex-AmB against axenic parasite cultures}

L. infantum ( 3 days culture) and L. amazonensis ( 5 days culture) axenic promastigotes, at a late exponential phase of growth, were seeded in 96-well plates using complete RPMI medium or complete Schneider's Insect medium, respectively, at a density of $3 \times 10^{5}$ cells/well. Then, increasing concentrations of free-AmB, Dex FD and SD and Dex-AmB FD and SD $(0.0029,0.0059,0.0117,0.0234,0.0469,0.0938,0.1875,0.375$ 
and $0.75 \mu \mathrm{M}$ ), previously sterilized by ethylene oxide for $15 \mathrm{~h}$ at $53^{\circ} \mathrm{C} \pm$ $1{ }^{\circ} \mathrm{C}$, were added to the wells and the plates were incubated for $24 \mathrm{~h}$ at $26^{\circ} \mathrm{C}$. Parasite viability was assessed by resazurin assay. Briefly, $20 \mu \mathrm{L}$ of a $2.5 \mathrm{mM}$ resazurin solution freshly prepared in phosphate buffer saline $\mathrm{pH} 7.4$ and filtered using sterile $0.22 \mu \mathrm{m}$ pore filters was added to each well and then the plates were incubated for $24 \mathrm{~h}$ at $26^{\circ} \mathrm{C}$. Fluorescence intensity was measured $\left(\lambda_{\text {ex }} 560 / \lambda_{\mathrm{em}} 590\right)$ in a SpectraMAX GeminiXS microplate reader (Molecular Devices LLC, California, USA). The results were expressed as the mean percentage \pm SD of viable parasites relatively to control condition (considered as $100 \%$ viability). The half maximal inhibitory concentration $\left(\mathrm{IC}_{50}\right)$ was calculated by nonlinear regression analysis using the GraphPad Prism software. The assay was performed in triplicate at least three times.

\subsubsection{Cell lines and cell culture}

Mouse fibroblast L929 cell line was purchased from American Type Culture Collection (ATCC, Virginia, USA), Human brain microvascular endothelial cell line (HBMEC) was kindly provided by Dr. Rui M. Reis (ICVS/3B's-PT Government Associate Laboratory, Braga, Portugal and Barretos Cancer Hospital, São Paulo, Brazil) and Human embryonic kidney (HEK293T) cell line was kindly provided by Dr. Tiago dos Santos (i3S - Instituto de Investigação e Inovação em Saúde, Universidade do Porto, Portugal; INEB - Instituto de Engenharia Biomédica, Universidade do Porto, Portugal). Both cell lines were maintained in Dulbecco's modified essential medium (DMEM) supplemented with $10 \%(\mathrm{v} / \mathrm{v})$ iFBS and $1 \%$ (v/v) Penicillin (10,000 U/mL)-Streptomycin $(10,000 \mu \mathrm{g} / \mathrm{mL})$ (complete DMEM medium) at $37{ }^{\circ} \mathrm{C}$ and $5 \% \mathrm{CO}_{2}$.

\subsubsection{Bone marrow-derived macrophages (BMM $\Phi)$ differentiation}

Mouse bone marrow cells were differentiated in vitro according to previous reported methods with some modifications [28]. C57BL/6 mice were anesthetized using a $\mathrm{CO}_{2}$ chamber and euthanized by cervical dislocation. Femurs and tibias were removed and cleaned in aseptic conditions. Bones were disconnected by the articulations and then flushed using complete DMEM medium. The obtained cell suspension of bone marrow cells was centrifuged $(300 \times \mathrm{g}, 10 \mathrm{~min})$ and the pellet re-suspended in $15 \mathrm{~mL}$ of complete DMEM medium supplemented with 20\% (v/v) L929-cell conditioned medium (LCCM) as source of macrophage colony-stimulating factor (M-CSF). Bone marrow was cultured overnight at $37^{\circ} \mathrm{C}$ in a $5 \% \mathrm{CO}_{2}$ atmosphere. The non-adherent cells were collected with warm complete DMEM medium supplemented with $20 \%$ $(\mathrm{v} / \mathrm{v}) \mathrm{LCCM}$, plated in a 96-well plate and incubated once again at $37^{\circ} \mathrm{C}$ in a $5 \% \mathrm{CO}_{2}$ atmosphere. On the 4 th and 7 th days the half of the medium was renewed and on the 10th day cells became fully differentiated into macrophages.

\subsubsection{Cell viability assay}

The cytotoxic effect of the nanocomplexes was assessed in BMМФ primary cells and in HEK293T, L929 and HBMEC cell lines using a standard resazurin assay, as described elsewhere [1]. Briefly, a monolayer of BMMФ (at a density of $3 \times 10^{4}$ cells/well), HEK293T and L929 (at a density of $1 \times 10^{4}$ cells/well) and HBMEC (at a density of $5 \times 10^{3}$ cells/well) were incubated for $24 \mathrm{~h}\left(37^{\circ} \mathrm{C}\right.$ in a $5 \% \mathrm{CO}_{2}$ atmosphere) with increasing concentrations of free-AmB, Dex FD or SD and Dex-AmB FD or SD (0.78, $1.17,1.76,2.63,3.95,5.93,8.89,13.33$ and $20 \mu \mathrm{M}$ ), previously sterilized by ethylene oxide for $15 \mathrm{~h}$ at $53{ }^{\circ} \mathrm{C} \pm 1{ }^{\circ} \mathrm{C}$. After the incubation period, $10 \%(\mathrm{v} / \mathrm{v})$ of a $2.5 \mathrm{mM}$ resazurin solution was added to each well and the cells were incubated at $37{ }^{\circ} \mathrm{C}$ in a $5 \% \mathrm{CO}_{2}$ atmosphere for another $4 \mathrm{~h}$. Fluorescence was measured ( $\left.\lambda_{\text {ex }} 560 / \lambda_{\text {em }} 590\right)$ in a SpectraMAX GeminiXS microplate reader (Molecular Devices LLC, California, USA). Results were expressed as the mean percentage \pm SD of viable cells relatively to control condition (considered as $100 \%$ viability). The concentration of a drug that kills half the BMMФ cells $\left(\mathrm{CC}_{50}\right)$ was calculated by nonlinear regression analysis using the GraphPad Prism software. The assay was performed in triplicate at least three times.

\subsubsection{Hemolysis assay}

Blood was obtained from a healthy dog after owners' agreement and collected to an EDTA tube. Whole blood was centrifuged (10 min at $1200 \times g, 4{ }^{\circ} \mathrm{C}$ ) and the supernatant and buffy coat were discarded. Red blood cells (RBCs) were resuspended in isotonic PBS pH 7.4. The purified RBCs were counted using a Neubauer chamber and diluted to a concentration of $1 \times 10^{8}$ cells $/ \mathrm{mL}$. Afterwards, $450 \mu \mathrm{L}$ of RBCs were placed in a 48-well plate containing $50 \mu \mathrm{L}$ of AmB, Dex FD or SD, DexAmB FD or SD (final concentrations of 32, 16, 8, 4, 2 and $1 \mu \mathrm{M}$ ) and the plates were incubated for $30 \mathrm{~min}$ at $37{ }^{\circ} \mathrm{C}$ under agitation (120 rpm). After incubation, solutions were collected to eppendorfs and centrifuged ( $10 \mathrm{~min}$ at $1200 \times \mathrm{g}, 4^{\circ} \mathrm{C}$ ). The supernatants were collected for analysis of the extent of hemolysis by reading the absorption of the hemoglobin at $540 \mathrm{~nm}$. Results were expressed as a percentage of hemolysis with respect to the amount of hemoglobin released in the presence of $1 \%$ of triton X-100, which was taken as measure of complete (100\%) lysis (Eq. (3)).

Hemolysis $(\%)=\frac{A b s s-A b s b}{A b s t-A b s b}$

Abss is the absorbance of the sample, Absb is the average absorbance of the negative control, and Abst is the average absorbance of the lysed samples. The assay was performed in triplicate at least three times.

\subsubsection{Anti-Leishmania activity of the Dex-AmB nanocomplexes against intramacrophagic amastigotes}

ВММФ were seeded in 96-well plates, at a cell density of $2 \times 10^{5}$ cells $/ \mathrm{mL}$. Subsequently, ВММФ were infected with $L$. infantum amastigotes at a multiplicity of infection (MOI) of 10 for $6 \mathrm{~h}$. After the infection period, non-internalized parasites were removed with complete DMEM medium and infected cells were incubated for an additional $24 \mathrm{~h}$. Increasing concentrations of free-AmB, Dex FD or SD, DexAmB FD or SD (0.0039, 0.0078, 0.0156, 0.03125, 0.0625, 0.125, 0.25, $0.5,1 \mu \mathrm{M}$ ) were added to the respective wells. After $24 \mathrm{~h}$ of incubation, cell monolayers were fixed with $4 \%(\mathrm{w} / \mathrm{v})$ paraformaldehyde, permeabilized with $0.1 \%(\mathrm{v} / \mathrm{v})$ Triton X-100 for $30 \mathrm{~min}$ at room temperature and then stained with $4^{\prime}$,6-diamidino-2-phenylindole (DAPI) and with high-content screening (HCS) CellMask ${ }^{\mathrm{TM}}$ Deep Red stain, which labels parasite DNA and macrophages nucleus and cytoplasm, respectively. The number of infected cells in each condition was determined in an IN Cell Analyzer 2000 microscope (GE Healthcare, Illinois, USA) as described by Gomes-Alves et al. [26]. Anti-Leishmania activity results were expressed as the mean percentage \pm SD of infected cells relatively to the control. The $\mathrm{IC}_{50}$ concentration was calculated by nonlinear regression analysis using the GraphPad Prism software. The assay was performed in triplicate at least three times.

\subsection{Statistics}

Statistical analysis was performed using GraphPad Prism 5 (GraphPad Software, San Diego, CA, USA). Results were expressed as mean \pm SD. Two-way ANOVA followed by Bonferroni's multiple comparison post-test was performed wherever appropriate. Significance was considered as $p<0.05$.

\section{Results and discussion}

\subsection{Production and characterization of Dex-AmB nanocomplexes}

The prepared nanocomplexes were firstly characterized in terms of size, zeta potential and morphology. Dex-AmB FD and Dex-AmB SD presented a mean diameter of $214.2 \pm 17.0$ and $347.5 \pm 36.1 \mathrm{~nm}$, respectively (Table 1). Size of nanomedicines is important as it influences biodistribution and clearance. Small particles $(<5-10 \mathrm{~nm})$ tend to have a reduced circulation time since they are rapidly cleared from 
Table 1

Average size, distribution and zeta potential of the Dex-AmB nanocomplexes dissolved in $\mathrm{dH}_{2} \mathrm{O}$. Results are expressed as mean $\pm \mathrm{SD}$.

\begin{tabular}{cccccc}
\hline & & \multicolumn{3}{c}{ Size distribution } & \multirow{3}{*}{$\begin{array}{c}\text { p potential } \\
(\mathrm{mV})\end{array}$} \\
\cline { 3 - 5 } Nanocomplex & Size $(\mathrm{nm})$ & $\mathbf{D 1 0}$ & D50 & D90 & \\
\hline \multirow{2}{*}{ Dex-AmB FD } & $214.2 \pm$ & $113.4 \pm$ & $193.5 \pm$ & $343.1 \pm$ & $-13.67 \pm$ \\
& 17.0 & 9.3 & 14.4 & 40.5 & 1.72 \\
Dex-AmB SD & $347.5 \pm$ & $155.0 \pm$ & $317.3 \pm$ & $577.3 \pm$ & $-1.90 \pm$ \\
& 36.1 & 24.5 & 39.1 & 51.1 & 0.21 \\
\hline
\end{tabular}

circulation through extravasation or renal clearance [29], while particles from 100 to $200 \mathrm{~nm}$ have been associated with increased phagocytosis by Leishmania-infected macrophages [30]. Additionally, some studies refer that nanoparticles larger than $200 \mathrm{~nm}$ are effective in targeting infected macrophages [31]. Table 1 shows that the produced material is polydisperse, presenting a wide size distribution. The size of the nanocomplexes may be considered safe both for intralesional or intravenous injection, since capillary blockage and embolism is only promoted by particles with size $>5 \mu \mathrm{m}$ [32]; it has been reported that Fungizone ${ }^{\circledR}$, which consist of particles with a mean size of $3310 \mathrm{~nm}$ [33], can be applied without any harmful effects [34].

The morphology of Dex-AmB FD and Dex-AMB SD was examined by Cryo-SEM (Fig. 1A and B) and SEM (Fig. 1C and D) (SEM analysis was only possible to perform with Dex-AmB SD powder). As shown in Fig. $1 A$ and $B$, when in aqueous solution Dex-AmB FD and Dex-AMB SD nanocomplexes have a spherical morphology, bearing a size consistent with the previously referred size distribution analysis. This result proves also that the rehydrated nanocomplexes are colloidally stable, as no aggregates are observed. Moreover, Dex-AmB SD powder analyzed by SEM (Fig. 1C and D) showed mostly spherical particles, with some concavities (possible due to collapse upon drying), smooth and non-porous surface. Similar results were obtained in another study where cyclodextrin-AmB spherical amorphous solid microparticles were prepared by spray drying [35]. The zeta potential of the DexAmB FD and Dex-AmB SD nanocomplexes was found to be $-13.67 \pm$ 1.72 and $-1.90 \pm 0.21 \mathrm{mV}$, respectively (Table 1 ) whereas Dex, noncharged glucose oligomers, has a zeta potential of $-8.34 \pm 2.71 \mathrm{mV}$. Generally, when particles have a high surface charge $(>|30 \mathrm{mV}|)$, they repel each other, which favors stability. In the current case, although the particles observed by Cryo-SEM do not exhibit signs of aggregation, the low zeta potential may compromise the long-term colloidal stability in aqueous suspension. However, the dry formulation is easy to disperse in water and to prepare just before use, thus the long-term stability in aqueous suspension is not a pivotal feature.

The interaction of $\mathrm{AmB}$ with the polysaccharide was confirmed using different techniques, including FTIR, DSC, PXRD. Fig. 2 displays the FTIR spectra, in the range of $700-4000 \mathrm{~cm}^{-1}$, for AmB, Dex FD, Dex-AmB FD, Dex SD and Dex-AmB SD. Polysaccharide processing by freeze- or spray-dryer did not cause any structural changes since all characteristic peaks of the dextrin are observed. More specifically, a strong and prominent absorption band at about $3386.7 \mathrm{~cm}^{-1}$ is observed which can be assigned to $-\mathrm{OH}$ stretching vibrations. The stretching of $\mathrm{C}-\mathrm{H}$ group vibrations and the $-\mathrm{OH}$ bending vibration is observed at 2927.7 and $1639.3 \mathrm{~cm}^{-1}$, respectively and the absorption bands at 1157.2 and $1026.0 \mathrm{~cm}^{-1}$ can be assigned to the stretching vibrations of $\mathrm{C}-\mathrm{O}$ and $\mathrm{C}-\mathrm{C}$, and the bending mode of $\mathrm{C}-\mathrm{H}$ and stretching vibration of $\mathrm{C}-\mathrm{O}-\mathrm{C}[36,37]$. Regarding the spectra of AmB crystals, it is possible to notice the absorption bands at 1558.3 and $1689.5 \mathrm{~cm}^{-1}$ which corresponds to the $\mathrm{C}=\mathrm{C}$ stretching vibration from
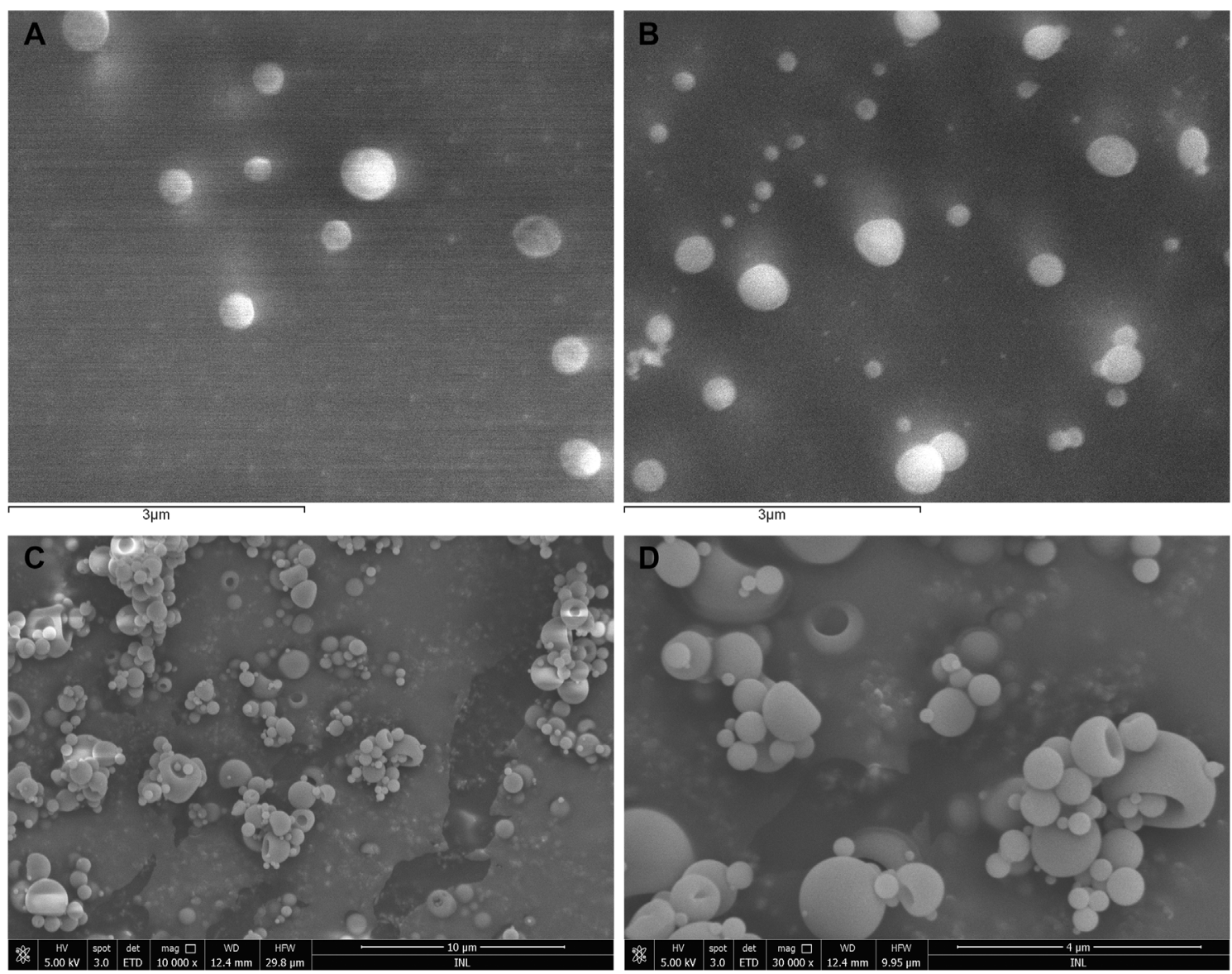

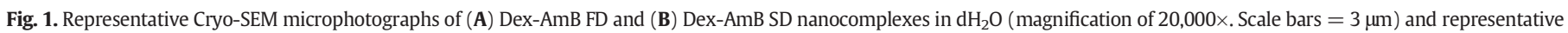
SEM microphotographs of Dex-AmB SD nanocomplex obtained at a magnification of $(\mathbf{C}) 10,000 \times($ scale bar $=10 \mu \mathrm{m})$ and $(\mathbf{D}) 300,000 \times($ scale bar $=4 \mu \mathrm{m})$. 
A
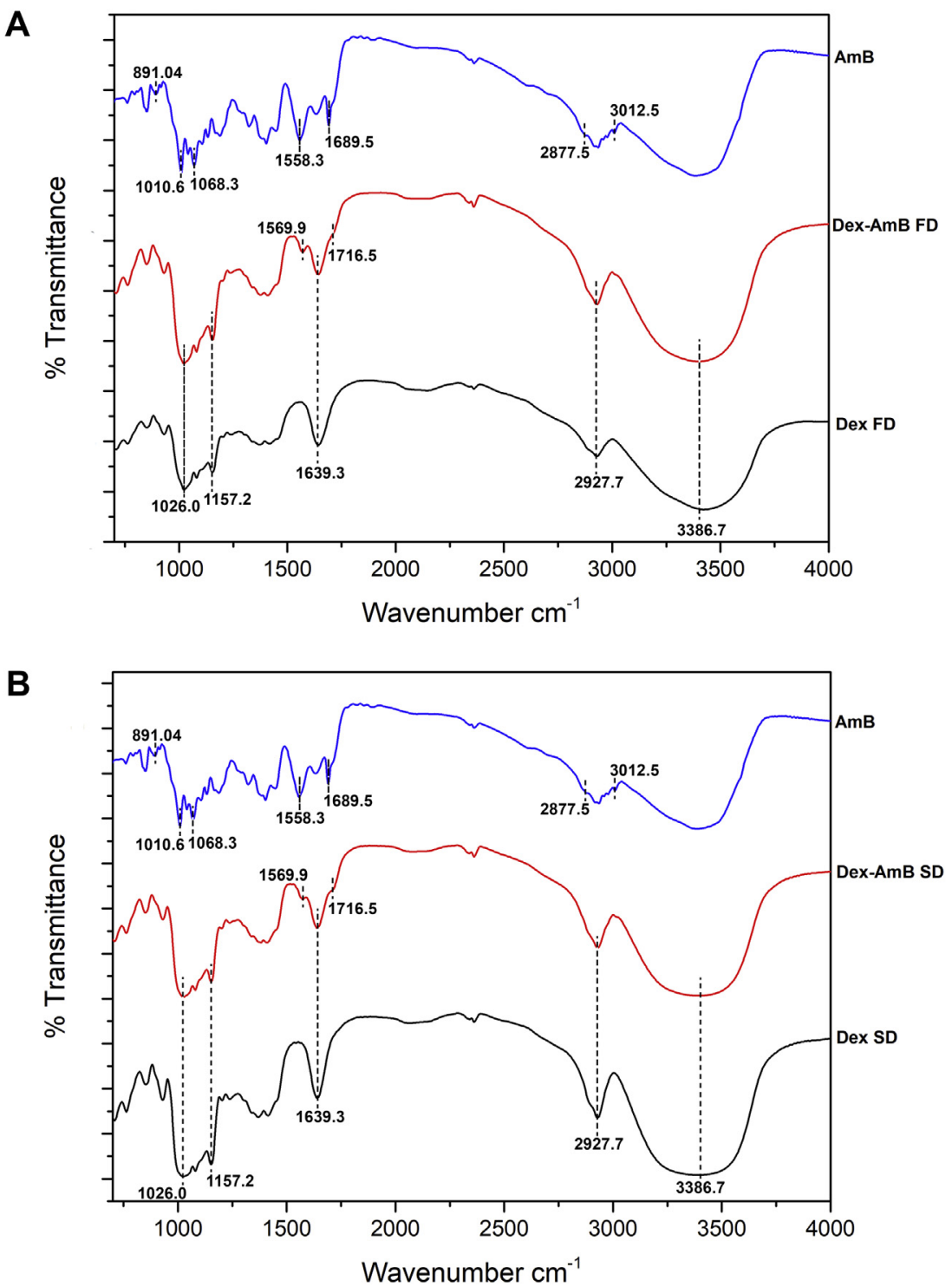

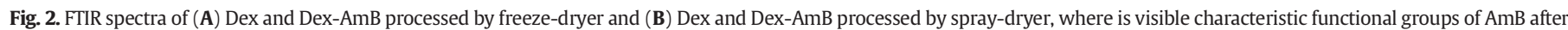
nanocomplexes production.

polyene and $\mathrm{C}=\mathrm{O}$ stretching vibration from the carbonyl ester group, respectively [38,39]. In addition, the peaks corresponding to $\mathrm{C}-\mathrm{H}$ stretching $\left(3012.5 \mathrm{~cm}^{-1}\right), \mathrm{CH}_{2}$ and $\mathrm{CH}_{3}$ symmetric stretching vibrations (2877.5 $\left.\mathrm{cm}^{-1}\right), \mathrm{C}-\mathrm{C}-\mathrm{H}$ (chromophore) bending as well as $\mathrm{CH}$ bending (trans-polyene) $\left(1010.6 \mathrm{~cm}^{-1}\right), \mathrm{C}=\mathrm{O}$ asymmetric stretching vibrations $\left(1068.4 \mathrm{~cm}^{-1}\right)$ and $\mathrm{C}-\mathrm{H}$ bending and $\mathrm{CH}_{3}$ rocking vibrations due to the $\mathrm{C}=\mathrm{O}$ asymmetric stretching vibrations $\left(891.04 \mathrm{~cm}^{-1}\right)$ are recognized [40,41]. FTIR spectra of Dex-AmB FD (Fig. 2A) and Dex-AmB SD (Fig. 2B) nanocomplexes are almost identical to their counterparts, but they also present characteristic peaks of the AmB. More specifically, both spectra feature the peaks corresponding to $\mathrm{C}=\mathrm{O}$ and $\mathrm{C}=\mathrm{C}$ stretching vibration, that suffer a slight shift from 1689.5 and $1558.3 \mathrm{~cm}^{-1}$ to 1716.5 and $1569.9 \mathrm{~cm}^{-1}$, respectively, probably due to the interaction with the polysaccharide. Similar results, where characteristic peaks of AmB were still visible in the FTIR analysis, were reported in the literature after drug conjugation to bovine serum albumin (BSA) [38] or encapsulation in glutaraldehyde cross-linked BSA microparticles [42], formation of AmB nano-assemblies with Aloe vera leaf extract [43] and conjugation of $A m B$ to oxidized sodium alginate [19] or oxidized polymannose [22].

Thermal studies are useful to ascertain the compatibility, stability and physical state of drugs conjugated to polymers or encapsulated in nanoparticles [36]. Fig. 3A and B shows the thermal behavior of $A m B$, Dex FD, Dex-AmB FD, Dex SD and Dex-AmB SD in the range $25-235^{\circ} \mathrm{C}$. As described in the literature, AmB was characterized by a double endothermic peak $[39,44]$, here determined at 165.9 (melting point of AmB crystal) and $199.5^{\circ} \mathrm{C}$ whereas Dex FD (Fig. 3A) and Dex SD (Fig. 3B) only showed a broad endothermic peak $\left(152.4{ }^{\circ} \mathrm{C}\right.$ and $162.2{ }^{\circ} \mathrm{C}$, respectively). The almost complete disappearance of the AmB endothermic peak was observed in the Dex-AmB FD (Fig. 3A) and Dex-AmB SD (Fig. 3B) nanocomplexes. Kim et al. [39] also showed that the incorporation of $\mathrm{AmB}$ in the cavities of the hydroxypropyl- $\gamma$ cyclodextrin (HP $\gamma C D$ ) promoted formation of an amorphous inclusion complex, as concluded from the lack of the AmB endothermic peak. Similar results were observed for conjugates of curcumin in dextrin [36] and of AmB in pegylated poly(D, L-lactide-co-glycolide) (PLGA) copolymer nanoparticles [45]. These results were further confirmed by PXRD (Fig. 3C and D). Free-AmB in pure state presents a crystalline lattice characterized by sharp peaks at diffraction angles of $2 \theta=14.06$, $17.26,18.4$, and 21.7, as reported elsewhere [39]. In the presence of the polysaccharide, after freeze (Fig. 3C) or spray dying (Fig. 3D), amorphization of the AmB is observed as characterized by the loss of the drug characteristic peaks, being only visible a large diffraction peak as in the processed free-polysaccharide. Similar results were 
A

C

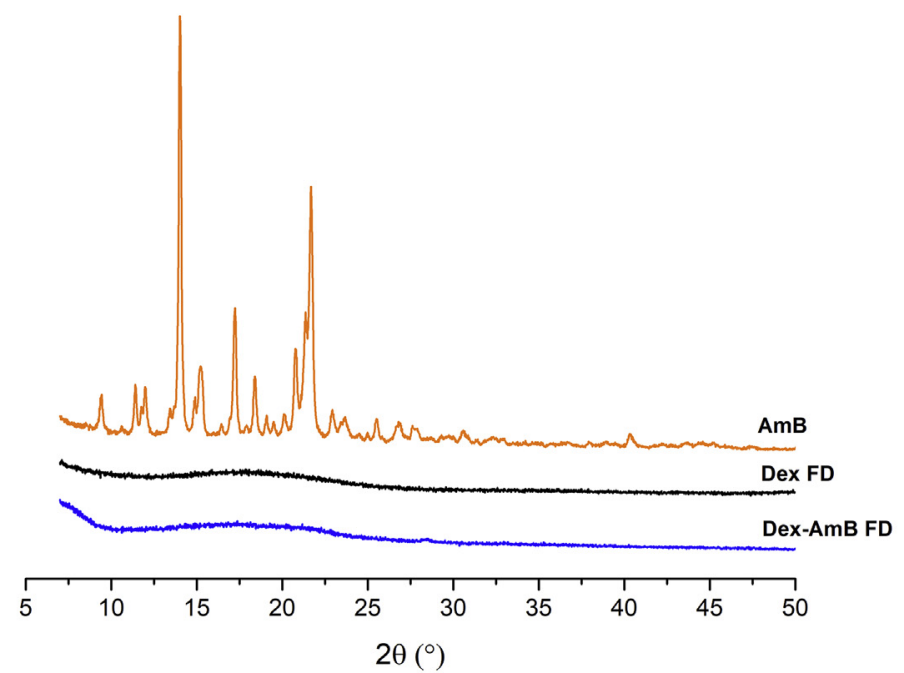

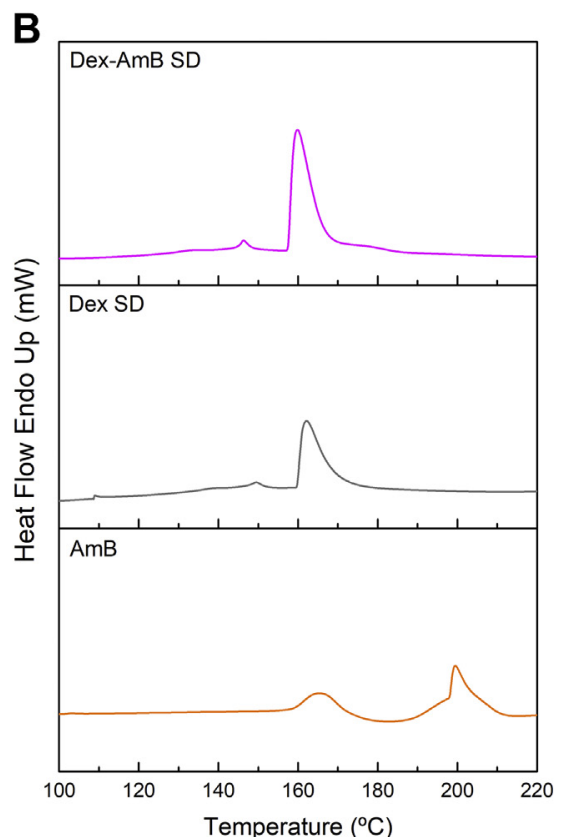

D

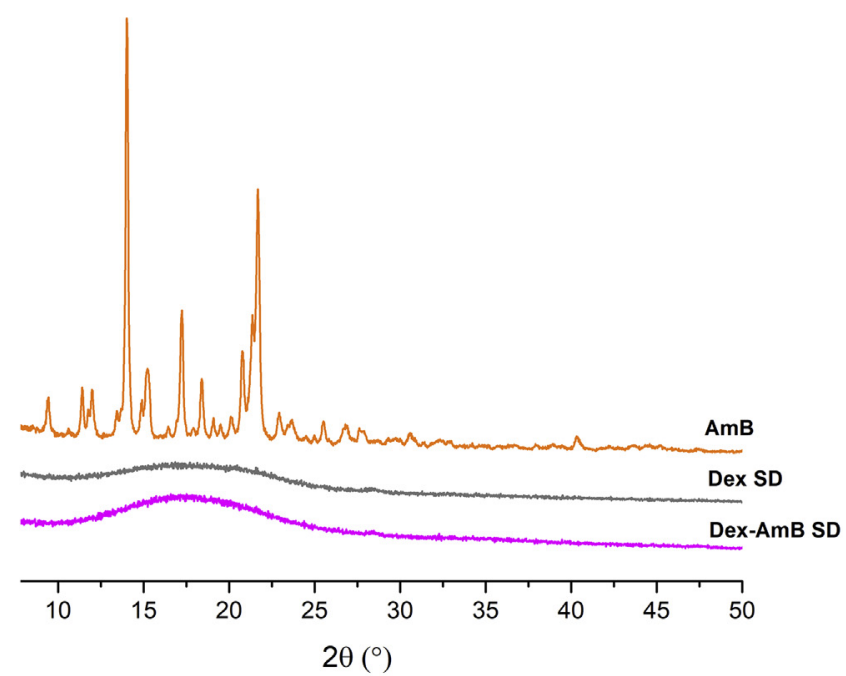

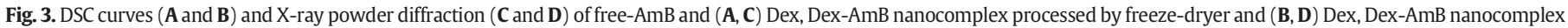
processed by spray-dryer.

reported in the literature where amorphous AmB nanoparticles were successfully prepared through liquid antisolvent precipitation followed by freeze-drying [46] and where AmB was encapsulated in polymeric micelle of PLGA grafted-dextran copolymer [47] or incorporated in the cavities of HP $\gamma C D$ [39]. Thus, we conclude that an amorphous inclusion complex between the AmB and the dextrin chain networks was formed.

\subsection{Spectroscopic analysis of Dex-AmB nanocomplexes}

UV-vis spectroscopy was used to investigate the aggregation state of the $\mathrm{AmB}$ in its free form or in the nanocomplexes, after dispersion in $\mathrm{dH}_{2} \mathrm{O}$. It is known that the AmB aggregation state is a key factor with direct impact on its cytotoxicity and activity $[48,49]$. In solution, AmB may exists in three forms: i) monomers, which is the soluble form, that normally associates with ergosterol in fungal and protozoan cell membranes; ii) oligomers (small soluble water aggregates; e.g. Fungizone $\left.{ }^{\circledR}\right)$, which can form pores in cholesterol-containing membranes, leading to toxicity towards host cells [50]; and iii) poly- aggregates [18,43], which is associated to lower in vitro and in vivo toxicity [51,52]. The UV-vis spectrum of AmB is very sensitive to aggregation and characterized by four well-separated bands when in a monomeric state [53,54]. Moreover, the spectral modifications induced by the extent of $A m B$ aggregation can be represented by the ratio of the first to the fourth peaks, being a value of $<1$ characteristic of the monomeric form [38] and a value of $>2$ to a supper-aggregated form [43]. Our results, showing four well-defined peaks at approximately 349 (I), 369 (II), 387 (III) and $408 \mathrm{~nm}$ (IV), indicates that free-AMB exists in monomeric form when firstly dissolved in $0.1 \mathrm{M}$ borate buffer $\mathrm{pH} 11$ and then diluted in $\mathrm{dH}_{2} \mathrm{O}$ (Fig. 4). Furthermore, the peak I/IV $\left(\mathrm{A}_{349} / \mathrm{A}_{408}\right)$ ratio for the $\mathrm{AmB}$ dispersed in the aqueous solution was 0.4 , confirming its presence in monomeric form. This value is similar to the one obtained in a study where AmB was dissolved in 50\% methanol [54]. The dissolution of the Dex-AmB FD nanocomplex (Fig. $4-\mathrm{B}$ ) in $\mathrm{dH}_{2} \mathrm{O}$ promoted a hypsochromic shift of the peak I to lower wavelength $(335 \mathrm{~nm})$, meaning that the $\mathrm{AmB}$ in the nanocomplex acquired a water-soluble aggregated form. In the case of the Dex-AmB SD nanocomplex (Fig. 4 - C), 


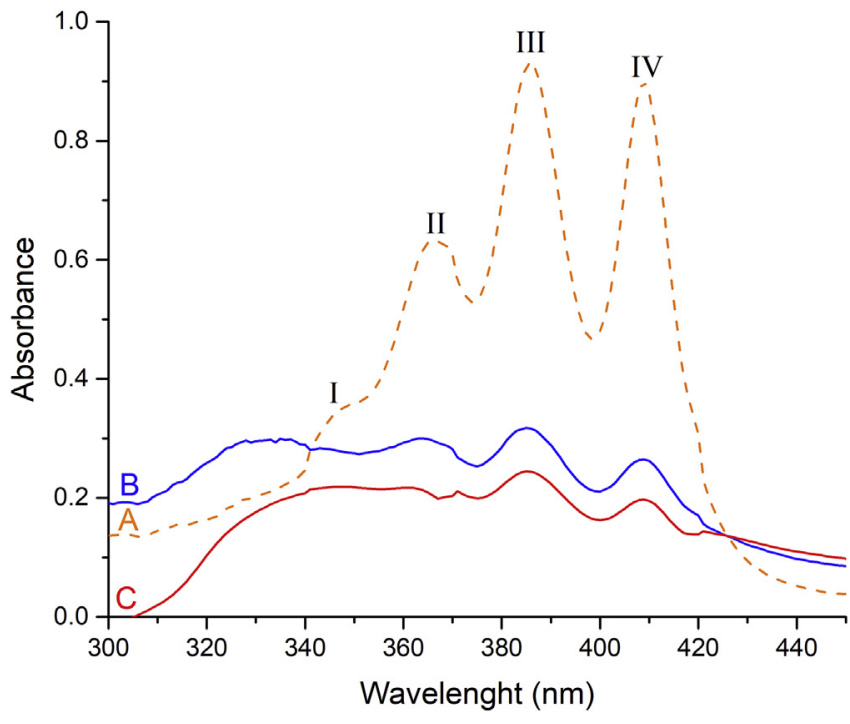

Fig. 4. Study of AmB aggregation state by UV-vis spectroscopy. Changes in the characteristic absorption peaks of $\mathrm{AmB}$ (at a final concentration of $10 \mu \mathrm{M}$ ) in $(\mathbf{A}$ ) its free form (dissolved in $0.1 \mathrm{M}$ borate buffer $\mathrm{pH} 11$ and diluted in dH2O), (B) Dex-AmB FD nanocomplex and (C) Dex-AmB SD nanocomplex (both dissolved and diluted in $\mathrm{dH}_{2} \mathrm{O}$ ).

even though the hypsochromic shift of the peak I is not prominent, we concluded that the AmB in this material is also present in a watersoluble aggregated form, since the UV-vis spectra of both nanocomplexes are very similar. As referred above, AmB is highly toxic in its aggregated state, promoting cytotoxicity [50]. For instance, the highly toxic formulation Fungizone ${ }^{\circledR}$ dissolved in $5 \%$ dextrose has a value of 2.9 [55]. For Dex-AmB FD $\left(\mathrm{A}_{335} / \mathrm{A}_{409}\right)$ and Dex-AmB SD $\left(A_{348} / A_{409}\right)$ nanocomplexes the degree of $A m B$ aggregation appears not to be significant since the ratio values were 1.13 and 1.11 , respectively. Furthermore, the higher the aggregation ratio, the lower the amount of monomeric AmB present [53]. Therefore, our results enable us to assume the presence of monomeric $\mathrm{AmB}$ in the nanocomplex, which may attenuate the toxicity of the formulation.

\subsection{AmB quantification by HPLC-PDA-MS}

The nanocomplexes were analyzed by HPLC using double detection (PDA and MS). The overall yield values (\%) are listed in Table 2. For DexAmB FD, the overall yield (86.8\%) is within the values reported for other polysaccharide-AmB formulations (approximately 60-90\%) [8,18-21]. Considering the Dex-AmB SD nanocomplex, a lower yield was achieved (51.5\%), as commonly reported in the development of spray dried pharmaceuticals at the small scale used, regardless the improvements achieved in the development of a nano spray dryer (final product yields increased theoretically to $\pm 90 \%$ ) [56].

While using spectrophotometry to assess the concentration of AmB, as it is widely used in the literature $[8,14,20,57]$, we noticed some lack of reproducibility and consistency, probably due to differences on the aggregation state. Therefore, an HPLC method was developed using a Kinetex® ${ }^{\circledR} 18$ column. For the analyzed mass range of $923-925 \mathrm{~m} / \mathrm{z}$ and wavelength of 387 and $408 \mathrm{~nm}$, linear regression in the range of concentrations used ( $2-120 \mu \mathrm{g} / \mathrm{mL}$ and $0.5-20 \mu \mathrm{g} / \mathrm{mL}$, respectively), with $\mathrm{r}^{2}$ between 0.9983 and 0.9991 was obtained. The AmB content (\% $\mathrm{w} / \mathrm{w})$ and association efficiency (\%) in the produced nanocomplexes were estimated using both detection methods (Table 2). The AmB content results obtained are quite similar regardless of whether they are estimated using the UV or the MS detection method or of the type of processing method used in the nanocomplexes production. As expected, comparatively to the freeze-dried material, the association efficiency determined for the spray-dried material is slightly inferior, since the obtained yields are also lower.

In the literature reporting other polysaccharides- or synthetic polysaccharide-based AmB formulations, AmB contents of 34.4, 36.6 and $32.9 \%$ were described for a dextran-AmB conjugate (amine), dextran-AmB (imine) and dextran-AmB-ethanolamine (imine), respectively [17], 23\% and 26\% for a arabinogalactan-AmB conjugates [8], 20\% for a galactomannan-AmB conjugate [21], 19.51\% for AmBpolymannose conjugates [22] and $18 \%$ for polyglucose-AmB conjugates [23]. Similarly to our work, Mehenni et al. [35] produced in a spray dryer a poly- $\gamma$-cyclodextrin-based $\mathrm{AmB}$ microspheres with a drug content of $13.72 \%$. Even in works reporting AmB encapsulation in nanoparticles, the drug content and/or association efficiency are in some cases slightly superior or comparable to our results $[34,47,53,54,57,58]$.

In this study, a Dex/AmB mass ratio of $4: 1$ was tested. However, since the nanocomplex production process is extremely simple, without any type of encapsulation or chemical modification being performed, increasing the drug content could be easily achieved by changing the polysaccharide/drug mass ratio used at the beginning of the process. Furthermore, the use of the spectrophotometric methods for drug content determination, a technique widely used in many of the above referred works, should be performed with caution as AmB UV-vis spectra is very sensitive to modifications (as discussed in the Section 3.2). This spectral instability might influence the determined absorbance values decreasing the reproducibility and consistency of the method and accuracy of the drug content determination.

\subsection{In vitro cytotoxicity assays}

Before testing the Dex-AmB formulations as a potential leishmanicidal therapy approach, the associated intrinsic toxicity was assessed. Thus, the cytotoxic effect of the free-AmB and of the different formulations was assessed using a variety of cells. For this purpose, a primary culture of BMM $\Phi$ and a kidney-derived HEK293T cell line (because AmB is associated with nephrotoxicity [59]) were employed. Moreover, L929 (fibroblasts) and HBMEC (endothelial) cell lines were also used since the produced material is intended to be applied either directly to a cutaneous lesion or intravenously.

As seen in Fig. $5 \mathrm{~A}$ and $\mathrm{C}$, free-AmB was cytotoxic, in a dosedependent way, towards levels under the $70 \%$ threshold (usually regarded as denoting cytotoxic potential [60]) at concentrations between 1.76 and $2.63 \mu \mathrm{M}\left(\mathrm{CC}_{50}\right.$ of $2.060 \pm 0.186 \mu \mathrm{M}$; Fig. S1). Regarding the HEK293T cells (Fig. $5 \mathrm{~B}$ and D), free-AmB also promoted a dose-dependent reduction of the cell viability at concentrations between 8.89 and $13.33 \mu \mathrm{M}$. These results showing toxicity of $\mathrm{AmB}$ against the two types of cells described above are in agreement with the literature. Toxic effects of AmB against J774.2 or Raw 264.7 macrophage cell lines at concentrations above

Table 2

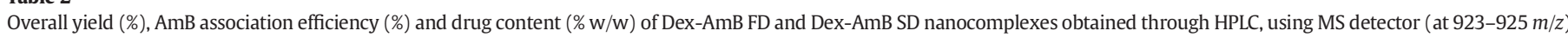
and UV detector (at 387 and $408 \mathrm{~nm}) .{ }^{*}(n=3)$.

\begin{tabular}{|c|c|c|c|c|c|c|c|}
\hline \multirow[b]{2}{*}{ Nanocomplex } & \multirow[b]{2}{*}{ Overall yield (\%) } & \multicolumn{3}{|c|}{ Drug content $(\%)^{*}$} & \multicolumn{3}{|c|}{ AmB association efficiency (\%)* } \\
\hline & & $\begin{array}{c}\text { MS } \\
(923-925 \mathrm{~m} / \mathrm{z})\end{array}$ & $\begin{array}{c}\text { UV } \\
(387 \mathrm{~nm})\end{array}$ & $\begin{array}{c}\text { UV } \\
(408 \mathrm{~nm})\end{array}$ & $\begin{array}{c}\text { MS } \\
(923-925 \mathrm{~m} / \mathrm{z})\end{array}$ & $\begin{array}{c}\mathrm{UV} \\
(387 \mathrm{~nm})\end{array}$ & $\begin{array}{c}\text { UV } \\
(408 \mathrm{~nm})\end{array}$ \\
\hline Dex-AmB FD & 86.8 & $14.68 \pm 2.16$ & $15.85 \pm 0.61$ & $14.85 \pm 0.54$ & $60 \pm 8.83$ & $64 \pm 2.38$ & $59 \pm 2.17$ \\
\hline Dex-AmB SD & 51.5 & $14.38 \pm 2.79$ & $16.22 \pm 0.83$ & $15.35 \pm 0.61$ & $33 \pm 5.24$ & $34 \pm 1.73$ & $32 \pm 1.27$ \\
\hline
\end{tabular}


A

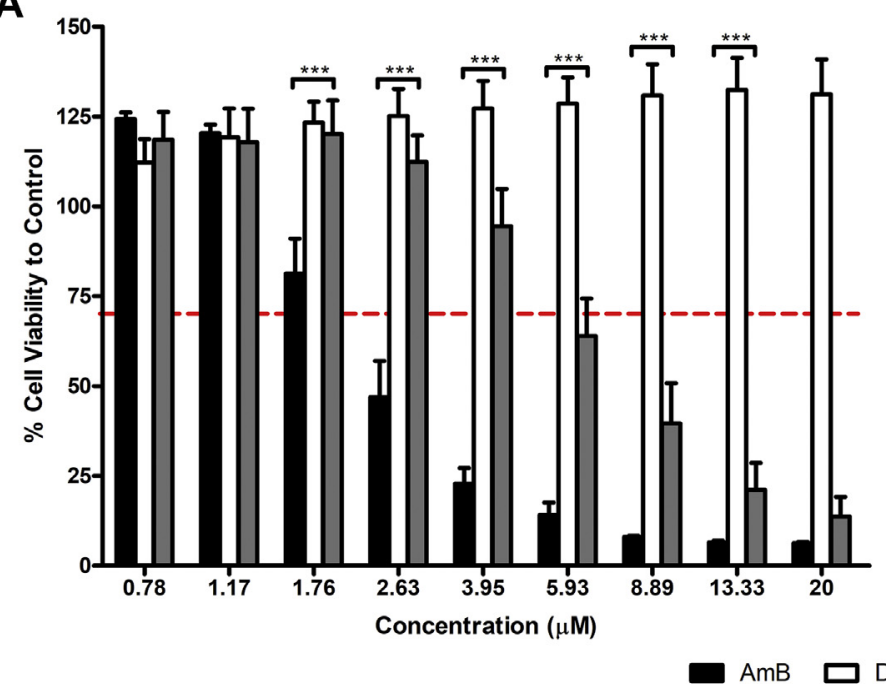

B

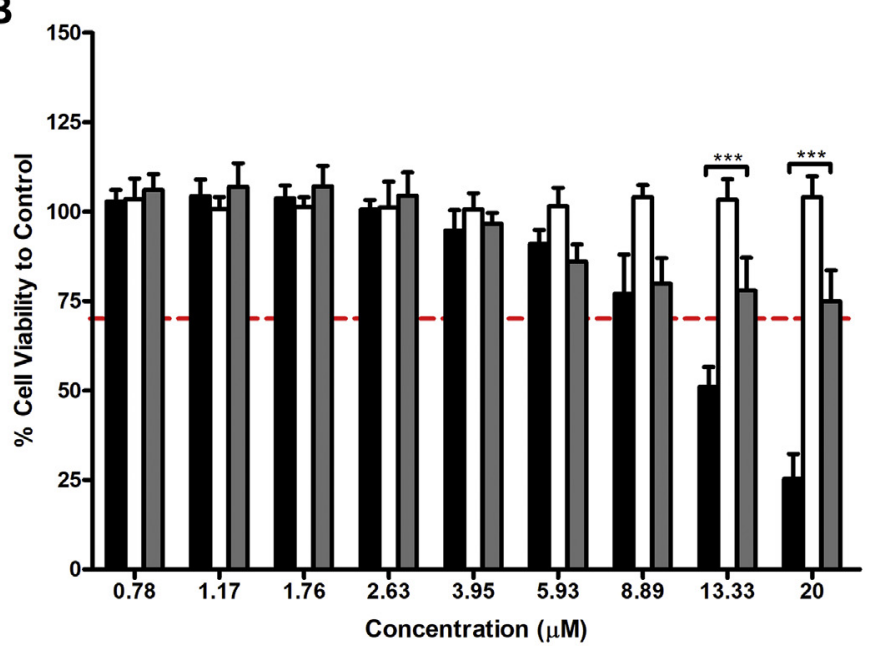

C

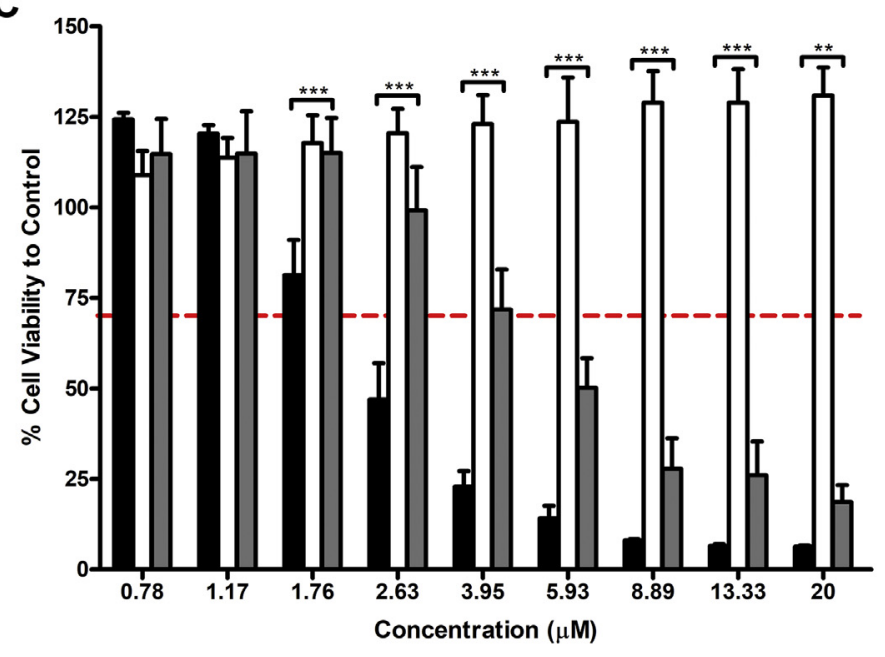

D

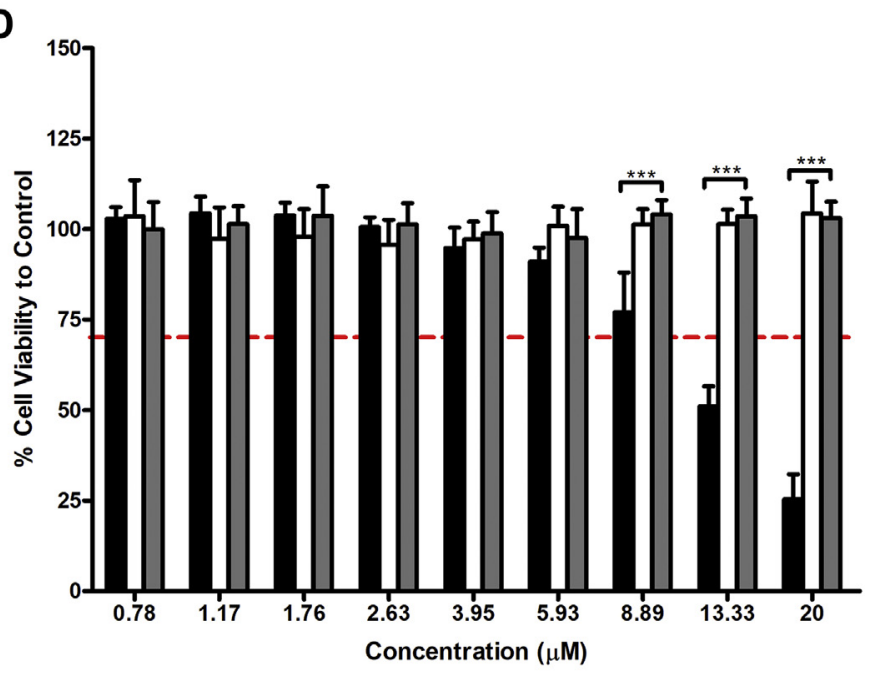

AmB $\square$ Dex SD

Dex-AmB SD

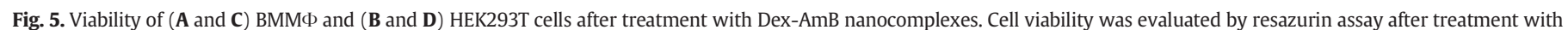

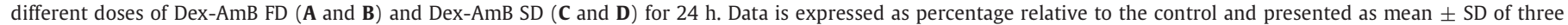

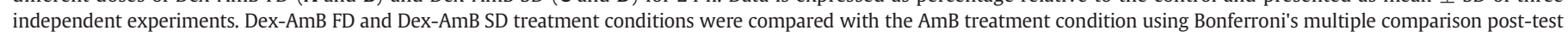
$\left({ }^{*} p<0.05,{ }^{* *} p<0.01\right.$ and $\left.{ }^{* * *} p<0.001\right)$.

$10 \mu \mathrm{g} / \mathrm{mL}[53,61]$, peritoneal macrophages at $23.1 \mu \mathrm{M}$ [62] or $7.6 \mu \mathrm{M}$ [63] and THP1 and ВMМФ cells at 19.8 and $21.8 \mu \mathrm{g} / \mathrm{mL}$, respectively [64] were described in the literature. Gurudevan et al. [38] showed that AmB at $15.6 \mu \mathrm{g} / \mathrm{mL}$ was highly cytotoxic against HEK293T cell line, promoting $>80 \%$ of cell death. In other studies, it was described that $7.8 \mu \mathrm{g} / \mathrm{mL}$ [22] or $10 \mu \mathrm{g} / \mathrm{mL}[19,23]$ of $\mathrm{AmB}$ promoted $50 \%$ or $90 \%$ of HEK293T cells death, respectively. Considering the variability in the doses of free-AmB that causes cytotoxicity, it is not surprising that our results differ somewhat from those reported. On the other hand, at the same concentrations, both Dex-AmB nanocomplexes were significantly less toxic against $В М М \Phi$ comparatively to the free drug. A reduction in cell viability to levels under the $70 \%$ threshold was only visible at concentrations between 3.95 and $5.93 \mu \mathrm{M}$ (Fig. $5 \mathrm{~A}$ and $\mathrm{C}$ ) $\left(\mathrm{CC}_{50}\right.$ of $5.77 \pm 1.27$ and $6.26 \pm 1.96 \mu \mathrm{M}$ for Dex-AmB FD and Dex-AmB SD, respectively; Fig. S1). Fig. 5 B and D shows that none of the Dex-AmB nanocomplexes promoted a reduction in HEK293T cell viability to levels under the $70 \%$ threshold even at the highest dose tested.

The reduction of AmB toxicity after inclusion or conjugation to polysaccharides or encapsulation into polymeric nanoparticles was also reported in the literature $[19,23,38,39,53]$. As expected, Dex was safe at all the tested concentrations (Fig. 5), since it is generally recognized as safe (GRAS) and previously developed dextrin-based formulations did not show cytotoxicity against ВМMФ and L929 cells [65,66]. Furthermore, the viability values above $100 \%$ seen for the Dex may be explained by the cell growth stimulant character of dextrin [67]. The AmB inclusion in the polysaccharide chain networks also allowed attenuating the toxicity of the drug against L929 and HBMEC cell lines (Fig. S2). The presented data appears to indicate that the Dex-AmB FD or DexAmB SD nanocomplexes are safe and reduce the toxicity of AmB. Furthermore, we speculate that in vivo the toxicity reduction may be even more significant as the nanocomplexes are expected to be swiftly internalized by the infected macrophages.

\subsection{Hemolytic activity}

Given that hemolysis is a severe side effect of AmB, we tested the hemolytic potential of Dex-AmB FD and Dex-AmB SD at various concentrations (ranging between 1 and $32 \mu \mathrm{M}$ ). According to ASTM E2524-08, materials resulting in over $5 \%$ hemolysis can be classified as hemolytic [68]. Different works in the literature show that pure AmB can lyse $>70 \%$ RBCs at a concentration of $10 \mu \mathrm{g} / \mathrm{mL}(10.8 \mu \mathrm{M})$ $[19,38,53]$. In this work, free-AmB was hemolytic towards dog RBCs at 
$4,8,16$ and $32 \mu \mathrm{M}$, leading to $97.6 \pm 7.8 \%$ of hemolysis at the higher concentration (Fig. 6).

The inclusion of $A m B$ into dextrin allowed a significant decrease in the hemolytic effect. In fact, Dex-AmB FD and Dex-AmB SD at $32 \mu \mathrm{M}$ promoted $61.5 \pm 10.8 \%$ (nearly 1.58 -fold less comparatively to freedrug) and $57.7 \pm 9.7 \%$ (nearly 1.69 -fold less comparatively to freedrug) of hemolysis, respectively, being the hemolytic activity negligible $(<5 \%)$ at $4 \mu \mathrm{M}$. This is consistent with findings of Zia et al. [43], who reported that a simple formation of AmB nano-assemblies with Aloe vera leaf extract decreased hemolysis of RBCs, even at $50 \mu \mathrm{g} / \mathrm{mL}$ AmB equivalents. In other studies, significant reduction of hemolysis to negligible levels was shown after AmB encapsulation into PLGA [57,69], chitosan [70] or polyglutamic acid [53] or conjugation to different polymers such as arabinogalactan [14], sodium alginate [19], gum arabic [18], dextran [17]. This outcome could be attributed to the shielding effect of the polysaccharide on $\mathrm{AmB}$ [19]. We tested also the hemolytic activity of the processed polysaccharide and, as expected, negligible hemolysis was observed even at the highest tested concentrations. Overall, our results suggest a wide safety margin of our nanocomplexes in bloodcontacting applications comparatively to the free-AmB and their suitability for intravenous administration.

\subsection{Anti-Leishmania activity against axenic promastigote and} intramacrophagic L. infantum amastigotes

Amphotericin B is active against both the extracellular promastigote and intracellular amastigote forms promoting the formation of ionic transmembrane channels, by the interaction with membrane ergosterol, leading to parasite death $[9,34]$. In this study, the in vitro leishmanicidal activity of AmB and Dex-AmB nanocomplexes was determined $24 \mathrm{~h}$ following exposure of $L$. infantum and L. amazonensis promastigotes or ВМMФ infected with $L$. infantum amastigotes to different concentrations of the above referred material. Regarding the axenic culture results (Fig. $7 \mathrm{~A}$ and $\mathrm{B}$ ), free-AmB was highly active against $L$. amazonensis and L. infantum, with $\mathrm{IC}_{50}$ values of $0.028 \pm 0.001$ and $0.023 \pm 0.010 \mu \mathrm{M}$, respectively. Some results reported in the literature, although referring to diverse species of Leishmania and presenting some variability, maintain the same tendency. Petri e Silva et al. [71] described an $\mathrm{IC}_{50}$ value of

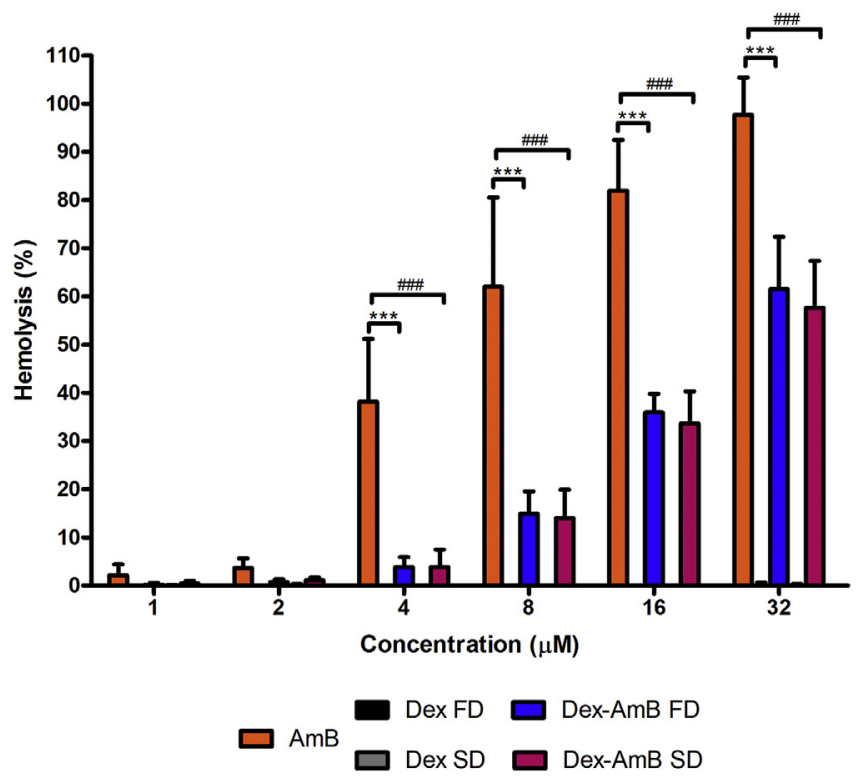

Fig. 6. Percentage of hemolysis caused by AmB and Dex-AmB nanocomplexes upon their interaction with dog RBCs with various concentrations (ranging between 1 and $32 \mu \mathrm{M}$ ) for $30 \mathrm{~min}$. Data is expressed as percentage relative to the control and presented as mean \pm SD of three independent experiments. Dex-AmB FD $\left(^{*}\right)$ and Dex-AmB SD (\#) treatment conditions were compared with the $A m B$ treatment condition using Bonferroni's multiple comparison post-test ( ${ }^{* * *}$ and \#\#\# $p<0.001$ ).
$0.22 \mu \mathrm{M}$ for $L$. infantum promastigotes after a $48 \mathrm{~h}$ exposure to AmB. In another study using $\mathrm{AmB}, \mathrm{IC}_{50}$ values from 0.01 to $0.27 \mu \mathrm{M}$ were reported, being the promastigote $L$. mexicana the least sensitive strain $(0.27 \mu \mathrm{M})$ and $L$. donovani the most sensitive strain $(0.012 \mu \mathrm{M})$ [72] of those tested, effect that was also reported in another study [23]. Chávez-Fumagalli et al. [73] reported $\mathrm{IC}_{50}$ values of 0.1 and $0.09 \mu \mathrm{g} / \mathrm{mL}$ for AmB-treated $L$. amazonensis and $L$. infantum, respectively. On the other hand, nanocomplexes also led to decreases in L. amazonensis and $L$. infantum viability, although at slightly higher concentrations comparatively to the free-drug. In fact, when comparing the $\mathrm{IC}_{50}$ of our nanocomplexes to free-AmB, it is noted that overall the latter has lower $\mathrm{IC}_{50}$. Dex-AmB FD and Dex-AmB SD presented $\mathrm{IC}_{50}$ values of $0.056 \pm 0.029$ and $0.096 \pm$ $0.050 \mu \mathrm{M}$, respectively, for L. amazonensis (Fig. $7 \mathrm{~A}$ ) and of $0.030 \pm$ 0.017 and $0.044 \pm 0.025 \mu \mathrm{M}$, respectively, for L. infantum (Fig. 7B). These differences were also observed in the literature for AmB conjugated to dextran [17], gum arabic [18], among others polymers [20,23]. The capacity to accurately test in vitro anti-Leishmania activity against Leishmania-infected $B M M \Phi$ is a critical point in the development of new formulations. For that, a protocol [26] for IN Cell Analyzer systems was recently developed and used here to obtain the dose-response curves for the L. infantum tested with the formulations, as seen in Fig. 7C. Results show that $A m B$ displayed high activity against intramacrophagic L. infantum parasites, as already reported in the literature $[26,71,74]$. Dex-AmB nanocomplexes had an activity similar to the free-drug, despite the slightly higher $\mathrm{IC}_{50}$ values $-0.017 \pm 0.009$ and $0.023 \pm 0.006 \mu \mathrm{M}$ for Dex-AmB FD and Dex-AmB SD, respectively - promoting a decrease in the amastigote parasite burden in the used concentration range. These results are consistent with the literature where reduced and unreduced AmB-AG conjugates were able to decrease the percentage of macrophages infected with L. infantum ( $\mathrm{ED}_{50}$ of $0.035 \mathrm{mg} / \mathrm{mL}$ and $0.027 \mathrm{mg} / \mathrm{mL}$, respectively) [75]. Importantly, the finding that Dex-AmB FD and Dex-AmB SD are approximately 2.47 - and 1.98 -fold more selective than free-drug is very promising (Table 3 ) and proves that the inclusion of AmB into the polysaccharide chain networks applying the simple processing method described in this work was able to improve the leishmanicidal potential of the drug. Dextrin did not show any significant effect, as expected, for all the tested concentrations in both assays.

\section{Conclusions}

The development of novel delivery systems for AmB to be used in leishmaniasis treatment, that improves the therapeutic index of this 'gold standard' drug, has been pursued over the years. In this work, we report a simple and economic formulation, where, unlike other polysaccharide-AmB formulations reported in the literature, no chemical modifications or solvents are required. Both nanocomplexes are easy to re-disperse in aqueous solutions and display leishmanicidal potential against axenic cultures of promastigotes and against intramacrophagic L. infantum amastigotes (selectivity index approximately 2.47- and 1.98 -fold superior to the free-AmB). All the presented results strongly suggest the Dex-AmB nanocomplexes have potential as a nanomedicine for Leishmaniasis treatment since, in addition to the affordability and simplicity of development, they are less toxic than other AmB based formulations.

\section{CRediT authorship contribution statement}

R. Silva-Carvalho:Investigation, Methodology, Formal analysis, Writing - original draft.J. Fidalgo:Methodology.K.R. Melo:Methodology.M.F. Queiroz:Methodology.S. Leal:Methodology.H.A. Rocha:Writing - review \& editing.T. Cruz:Methodology.P. Parpot:Methodology, Validation, Writing - review \& editing.A.M. Tomás:Validation, Writing - review \& editing.M. Gama:Conceptualization, Writing - review \& editing, Supervision. 
A

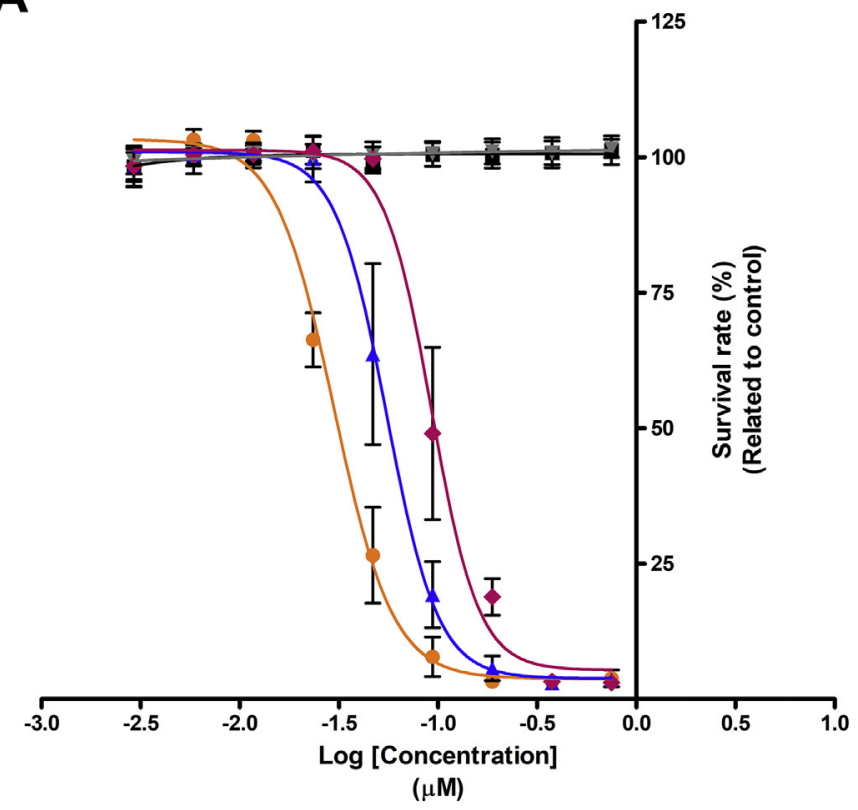

B

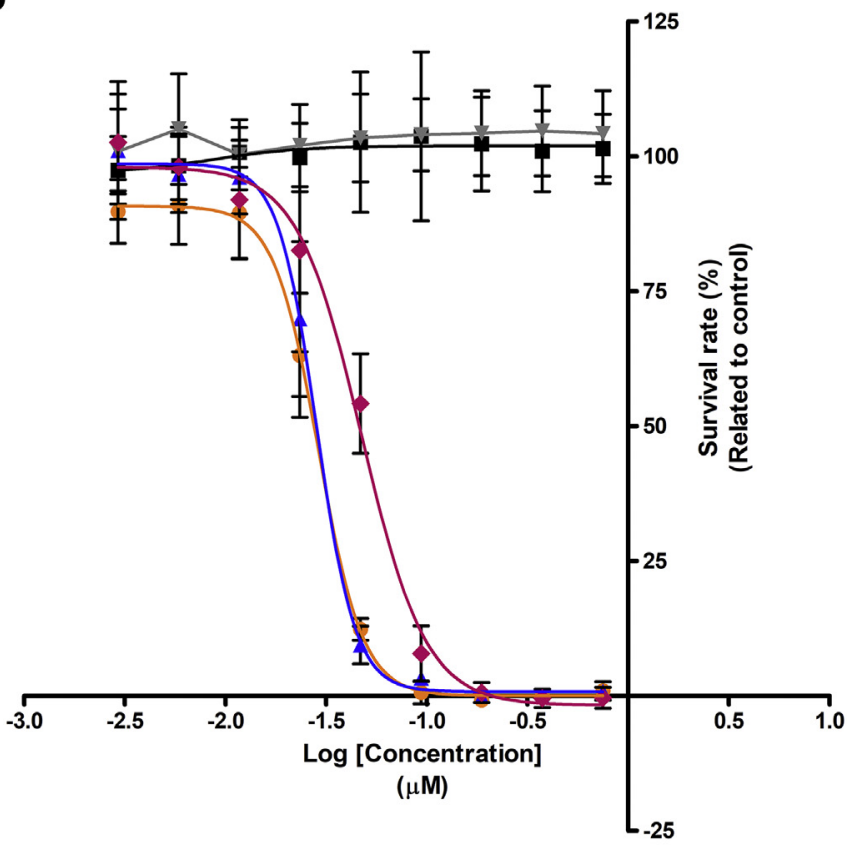

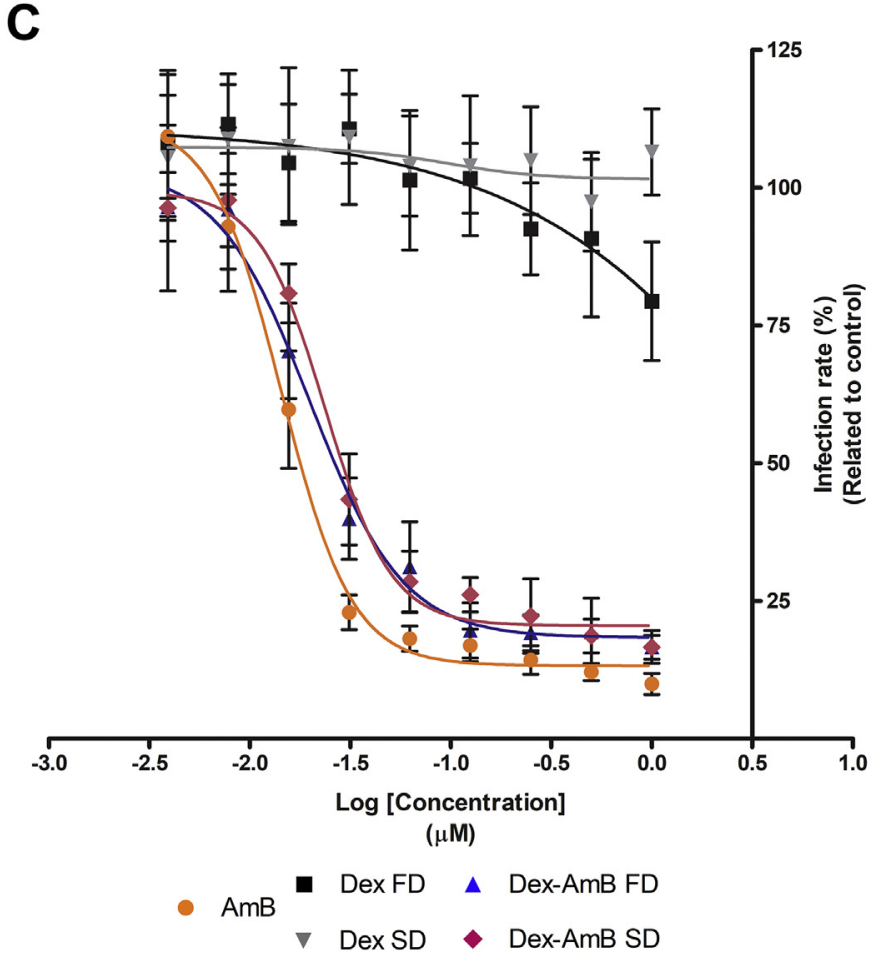

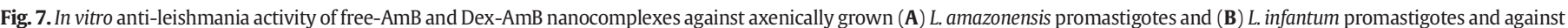

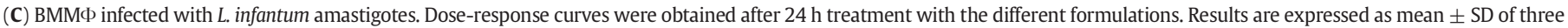
independent experiments.

Table 3

In vitro cytotoxicity against intracellular amastigotes and macrophages.

\begin{tabular}{lccc}
\hline Sample & $\begin{array}{c}\text { Amastigotes } \mathrm{IC}_{50} \\
(\mu \mathrm{M})\end{array}$ & $\begin{array}{c}\text { Macrophages } \mathrm{CC}_{50} \\
(\mu \mathrm{M})\end{array}$ & $\begin{array}{c}\text { Selectivity index } \\
(\mathrm{SI})^{\mathrm{a}}\end{array}$ \\
\hline AmB & $0.015 \pm 0.003$ & $2.060 \pm 0.190$ & 137.3 \\
Dex-AmB FD & $0.017 \pm 0.009$ & $5.769 \pm 1.273$ & 339.4 \\
Dex-AmB SD & $0.023 \pm 0.006$ & $6.263 \pm 1.963$ & 272.3 \\
\hline
\end{tabular}

Means \pm SD $(\mathrm{n}=3)$.

a $\mathrm{SI}$ (selectivity index) $=\mathrm{CC}_{50} / \mathrm{IC}_{50}$.

\section{Declaration of competing interest}

The authors declare no conflict of interest.

\section{Acknowledgements}

The authors would like to acknowledge Lorenzo Pastrana, Catarina Gonçalves and Ana Isabel Bourbon from the Food Processing group of International Iberian Nanotechnology Laboratory, for the use of Nano Spray dryer and SEM. 
This work was supported by the Portuguese Foundation for Science and Technology (FCT) under the scope of the strategic funding of UID/ BIO/04469/2019 and BioTecNorte operation (NORTE-01-0145-FEDER000004 - Underpinning Biotechnology to foster the north of Portugal bioeconomy and NORTE-01-0145-FEDER-000012 - Structured program on bioengineered therapies for Infectious diseases and tissue regeneration) funded by the European Regional Development Fund under the scope of Norte2020 - Programa Operacional Regional do Norte. Ricardo Silva-Carvalho gratefully acknowledge FCT for the granted scholarship (SFRH/BD/118880/2016). Karoline Rachel Melo and Moacir Fernandes Queiroz gratefully acknowledge Coordenação de Aperfeiçoamento de Pessoal de Nível Superior (CAPES), Brasil for the granted scholarship.

\section{Appendix A. Supplementary data}

Dose-response curves of the in vitro cytotoxic activity of free-AmB and Dex-AmB nanocomplexes against ВММ $\Phi$ after $24 \mathrm{~h}$ of treatment; Viability of (A and C) L929 and (B and D) HBMEC cells after treatment with different doses of Dex-AmB FD (A and B) and Dex-AmB SD (C and $\mathrm{D}$ ) for $24 \mathrm{~h}$. Supplementary data to this article can be found online at https://doi.org/10.1016/j.ijbiomac.2020.03.019.

\section{References}

[1] S. Vale-Costa, et al., Iron overload favors the elimination of Leishmania infantum from mouse tissues through interaction with reactive oxygen and nitrogen species, PLoS Negl. Trop. Dis. 7 (2) (2013), e2061.

[2] L. Kedzierski, Leishmaniasis vaccine: where are we today? J Glob Infect Dis 2 (2) (2010) 177-185.

[3] E. Torres-Guerrero, et al., Leishmaniasis: a review, F1000Res 6 (2017) 750.

[4] P. Desjeux, Leishmaniasis: current situation and new perspectives, Comp. Immunol. Microbiol. Infect. Dis. 27 (5) (2004) 305-318.

[5] A. Oryan, M. Akbari, Worldwide risk factors in leishmaniasis, Asian Pac J Trop Med 9 (10) (2016) 925-932.

[6] P. Kaye, P. Scott, Leishmaniasis: complexity at the host-pathogen interface, Nat Rev Microbiol 9 (8) (2011) 604-615.

[7] M. Olivier, Culprit within a culprit, Nature 471 (2011) 173.

[8] T. Ehrenfreund-Kleinman, et al., Synthesis and characterization of novel water soluble amphotericin B-arabinogalactan conjugates, Biomaterials 23 (5) (2002) 1327-1335.

[9] M. Baginski, et al., Interaction of amphotericin B and its selected derivatives with membranes: molecular modeling studies, Chem. Rec. 6 (6) (2006) 320-332.

[10] M. Baran, et al., Molecular modeling of amphotericin B-ergosterol primary complex in water II, Biophys. Chem. 141 (2) (2009) 162-168.

[11] C. Serafim, et al., Lipoamino acid-based micelles as promising delivery vehicles for monomeric amphotericin B, Int. J. Pharm. 497 (1-2) (2016) 23-35.

[12] J. Brajtburg, J. Bolard, Carrier effects on biological activity of amphotericin B, Clin. Microbiol. Rev. 9 (4) (1996) 512-531.

[13] R. Duncan, M.J. Vicent, Polymer therapeutics-prospects for 21st century: the end of the beginning, Adv. Drug Deliv. Rev. 65 (1) (2013) 60-70.

[14] T. Ehrenfreund-Kleinman, et al., Conjugation of amino-containing drugs to polysaccharides by tosylation: amphotericin B-arabinogalactan conjugates, Biomaterials 25 (15) (2004) 3049-3057.

[15] D.E. Ickowicz, et al., Activity, reduced toxicity, and scale-up synthesis of amphotericin B-conjugated polysaccharide, Biomacromolecules 15 (6) (2014) 2079-2089.

[16] S.P. Hudson, et al., Injectable in situ cross-linking hydrogels for local antifungal therapy, Biomaterials 31 (6) (2010) 1444-1452.

[17] M. Sokolsky-Papkov, et al., Impact of aldehyde content on amphotericin B-dextran imine conjugate toxicity, Biomacromolecules 7 (5) (2006) 1529-1535.

[18] K.K. Nishi, et al., Amphotericin B-gum arabic conjugates: synthesis, toxicity, bioavailability, and activities against Leishmania and fungi, Pharm. Res. 24 (5) (2007) 971-980.

[19] V. Ravichandran, A. Jayakrishnan, Synthesis and evaluation of anti-fungal activities of sodium alginate-amphotericin B conjugates, Int. J. Biol. Macromol. 108 (2018) $1101-1109$.

[20] G.P. Kothandaraman, et al., Anti-fungal and anti-leishmanial activities of pectinamphotericin B conjugates, J Drug Deliv Sci Technol 39 (2017) 1-7.

[21] S. Farber, et al., Galactomannan-amphotericin B conjugate: synthesis and biological activity, Polym. Adv. Technol. 22 (1) (2011) 119-125.

[22] A.P. Francis, et al., Synthetic polymannose as a drug carrier: synthesis, toxicity and anti-fungal activity of polymannose-amphotericin B conjugates, J Biomater Sci Polym Ed 29 (13) (2018) 1529-1548.

[23] V. Ravichandran, et al., Synthetic polysaccharides as drug carriers: synthesis of polyglucose-amphotericin B conjugates and in vitro evaluation of their anti-fungal and anti-leishmanial activities, J. Nanosci. Nanotechnol. 18 (4) (2018) 2405-2414.

[24] D.M. Silva, et al., Structural analysis of dextrins and characterization of dextrinbased biomedical hydrogels, Carbohydr. Polym. 114 (2014) 458-466.
[25] A.V. Delgado, et al., Measurement and interpretation of electrokinetic phenomena, J. Colloid Interface Sci. 309 (2) (2007) 194-224

[26] A.G. Gomes-Alves, et al., Development of an automated image analysis protocol for quantification of intracellular forms of Leishmania spp, PLoS One 13 (8) (2018), e0201747.

[27] D. Sereno, J.L. Lemesre, Axenically cultured amastigote forms as an in vitro model for investigation of antileishmanial agents, Antimicrob. Agents Chemother. 41 (5) (1997) 972-976.

[28] M.S. Gomes, et al., Engagement of Toll-like receptor 2 in mouse macrophages infected with Mycobacterium avium induces non-oxidative and TNF-independent anti-mycobacterial activity, Eur. J. Immunol. 38 (8) (2008) 2180-2189.

[29] S.V. Vinogradov, et al., Nanosized cationic hydrogels for drug delivery: preparation, properties and interactions with cells, Adv. Drug Deliv. Rev. 54 (1) (2002) 135-147.

[30] J.K. Vasir, et al., Nanosystems in drug targeting: opportunities and challenges, Curr. Nanosci. 1 (1) (2005) 47-64.

[31] R. Kalluru, et al., Poly(lactide-co-glycolide)-rifampicin nanoparticles efficiently clear Mycobacterium bovis BCG infection in macrophages and remain membrane-bound in phago-lysosomes, J. Cell Sci. 126 (Pt 14) (2013) 3043-3054.

[32] L. Wang, et al., Safety of nanosuspensions in drug delivery, Nanomedicine 13 (2) (2017) 455-469.

[33] C. Alvarez, et al., Reformulation of fungizone by PEG-DSPE micelles: deaggregation and detoxification of amphotericin B, Pharm. Res. 33 (9) (2016) 2098-2106.

[34] A. Abu Ammar, et al., Amphotericin B-loaded nanoparticles for local treatment of cutaneous leishmaniasis, Drug Deliv Transl Res 9 (1) (2019) 76-84.

[35] L. Mehenni, et al., Preparation and characterization of spherical amorphous solid dispersion with amphotericin B, Pharmaceutics 10 (4) (2018)

[36] B. Sun, et al., Linear dextrin as curcumin delivery system: effect of degree of polymerization on the functional stability of curcumin, Food Hydrocoll. 77 (2018) 911-920.

[37] X. Hu, et al., Synthesis and characterization of dextrin monosuccinate, Carbohydr. Polym. 97 (1) (2013) 111-115.

[38] S. Gurudevan, et al., Amphotericin B-albumin conjugates: synthesis, toxicity and anti-fungal activity, Eur. J. Pharm. Sci. 115 (2018) 167-174

[39] Y.T. Kim, et al., A thermosensitive vaginal gel formulation with HPgammaCD for the pH-dependent release and solubilization of amphotericin B, Eur. J. Pharm. Sci. 41 (2) (2010) 399-406

[40] M. Gagoś, M. Arczewska, FTIR spectroscopic study of molecular organization of the antibiotic amphotericin B in aqueous solution and in DPPC lipid monolayers containing the sterols cholesterol and ergosterol, Eur. Biophys. J. 41 (8) (2012) 663-673.

[41] G. Schwartzman, et al., Ambiguities in IR and X-ray characterization of amphotericin B, J. Pharm. Sci. 67 (3) (1978) 398-400.

[42] P.K. Angra, et al., Amphotericin B microspheres: a therapeutic approach to minimize toxicity while maintaining antifungal efficacy, J. Microencapsul. 26 (7) (2009) 580-587.

[43] Q. Zia, et al., Biomimetically engineered Amphotericin B nano-aggregates circumvent toxicity constraints and treat systemic fungal infection in experimental animals, Sci. Rep. 7 (1) (2017), 11873.

[44] L. Zhou, et al., Preparation, characterization, and evaluation of amphotericin Bloaded MPEG-PCL-g-PEI micelles for local treatment of oral Candida albicans, Int. J. Nanomedicine 12 (2017) 4269-4283.

[45] B.T. Al-Quadeib, et al., Stealth amphotericin B nanoparticles for oral drug delivery: in vitro optimization, Saudi Pharm J 23 (3) (2015) 290-302.

[46] Y. Zu, et al., Preparation and characterization of amorphous amphotericin B nanoparticles for oral administration through liquid antisolvent precipitation, Eur. J. Pharm. Sci. 53 (2014) 109-117.

[47] K.C. Choi, et al., Amphotericin B-incorporated polymeric micelles composed of poly (d,l-lactide-co-glycolide)/dextran graft copolymer, Int. J. Pharm. 355 (1-2) (2008) 224-230.

[48] J.A. Sanchez-Brunete, et al., Amphotericin B molecular organization as an essential factor to improve activity/toxicity ratio in the treatment of visceral leishmaniasis, J. Drug Target. 12 (7) (2004) 453-460.

[49] R. Espada, et al., In vivo distribution and therapeutic efficacy of a novel amphotericin B poly-aggregated formulation, J. Antimicrob. Chemother. 61 (5) (2008) $1125-1131$.

[50] M. Larabi, et al., New lipid formulation of amphotericin B: spectral and microscopic analysis, Biochim. Biophys. Acta 1664 (2) (2004) 172-181

[51] E.W. van Etten, et al., Mild heating of amphotericin B-desoxycholate: effects on ultrastructure, in vitro activity and toxicity, and therapeutic efficacy in severe candidiasis in leukopenic mice, Antimicrob. Agents Chemother. 44 (6) (2000) 1598-1603.

[52] C. Petit, et al., Activity of a heat-induced reformulation of amphotericin B deoxycholate (fungizone) against Leishmania donovani, Antimicrob. Agents Chemother. 43 (2) (1999) 390-392.

[53] Q. Zia, et al., Self-assembled amphotericin B-loaded polyglutamic acid nanoparticles: preparation, characterization and in vitro potential against Candida albicans, Int. J. Nanomedicine 10 (2015) 1769-1790.

[54] W. Tiyaboonchai, N. Limpeanchob, Formulation and characterization of amphotericin B-chitosan-dextran sulfate nanoparticles, Int. J. Pharm. 329 (1-2) (2007) 142-149.

[55] A.B. Mullen, et al., Comparison of the efficacies of various formulations of amphotericin B against murine visceral leishmaniasis, Antimicrob. Agents Chemother. 41 (10) (1997) 2089-2092.

[56] A. Faheem, Y. Haggag, Evaluation of nano spray drying as a method for drying and formulation of therapeutic peptides and proteins, Front. Pharmacol. 6 (140) (2015)

[57] H. Van de Ven, et al., PLGA nanoparticles and nanosuspensions with amphotericin B: potent in vitro and in vivo alternatives to Fungizone and AmBisome, J. Control. Release 161 (3) (2012) 795-803. 
ARTICLE IN PRESS

R. Silva-Carvalho et al. / International Journal of Biological Macromolecules 153 (2020) xxx

13

[58] D. Butani, et al., Topical amphotericin B solid lipid nanoparticles: design and development, Colloids Surf B Biointerfaces 139 (2016) 17-24

[59] G. Deray, Amphotericin B nephrotoxicity, J. Antimicrob. Chemother. 49 (Suppl. 1) (2002) 37-41.

[60] ISO10993-5:2009, Biological Evaluation of Medical Devices - Part 5: Tests for In Vitro Cytotoxicity, 2009.

[61] M.S. Espuelas, et al., Polymeric carriers for amphotericin B: in vitro activity, toxicity and therapeutic efficacy against systemic candidiasis in neutropenic mice, J. Antimicrob. Chemother. 52 (3) (2003) 419-427.

[62] A.M.A. Velásquez, et al., Efficacy of a binuclear cyclopalladated compound therapy for cutaneous leishmaniasis in the murine model of infection with Leishmania amazonensis and its inhibitory effect on topoisomerase 1B, Antimicrob. Agents Chemother. 61 (8) (2017)e00688-17.

[63] P.S. Lage, et al., Antileishmanial activity and evaluation of the mechanism of action of strychnobiflavone flavonoid isolated from Strychnos pseudoquina against Leishmania infantum, Parasitol. Res. 114 (12) (2015) 4625-4635.

[64] D. Barros, et al., Surface functionalization of polymeric nanospheres modulates macrophage activation: relevance in leishmaniasis therapy, Nanomedicine (Lond) 10 (3) (2015) 387-403.

[65] K. Alvani, et al., Use of carbohydrates, including dextrins, for oral delivery, Starke 63 (7) (2011) 424-431.

[66] C. Gonçalves, et al., Dextrin nanoparticles: studies on the interaction with murine macrophages and blood clearance, Colloids Surf B Biointerfaces 75 (2) (2010) 483-489.
[67] T. Asai, et al., In vitro biocompatibility of dextrin: the addition of a low concentration of dextrin in the medium promotes the cell activity of L929 mouse fibroblasts, Cel Biol. Int. 35 (6) (2011) 645-648.

[68] ASTME2524-08(2013), Standard Test Method for Analysis of Hemolytic Properties of Nanoparticles, ASTM International, West Conshohocken, PA, 2013.

[69] T.C. Moraes Moreira Carraro, et al., Assessment of in vitro antifungal efficacy and in vivo toxicity of Amphotericin B-loaded PLGA and PLGA-PEG blend nanoparticles, J Mycol Med 27 (4) (2017) 519-529.

[70] T.G. Ribeiro, et al., Novel targeting using nanoparticles: an approach to the development of an effective anti-leishmanial drug-delivery system, Int. J. Nanomedicine 9 (2014) 877-890.

[71] S.C. Petri e Silva, et al., Effects of nitro-heterocyclic derivatives against Leishmania (Leishmania) infantum promastigotes and intracellular amastigotes, Exp. Parasitol. 163 (2016) 68-75.

[72] P. Escobar, et al., Sensitivities of Leishmania species to hexadecylphosphocholine (miltefosine), ET-18-OCH(3) (edelfosine) and amphotericin B, Acta Trop. 81 (2) (2002) 151-157.

[73] M.A. Chavez-Fumagalli, et al., New delivery systems for amphotericin B applied to the improvement of leishmaniasis treatment, Rev. Soc. Bras. Med. Trop. 48 (3) (2015) 235-242.

[74] K. Seifert, et al., In vitro activity of anti-leishmanial drugs against Leishmania donovani is host cell dependent, J. Antimicrob. Chemother. 65 (3) (2010) 508-511.

[75] R. Falk, et al., A novel injectable water-soluble amphotericin B-arabinogalactan conjugate, Antimicrob. Agents Chemother. 43 (8) (1999) 1975-1981.

Please cite this article as: R. Silva-Carvalho, J. Fidalgo, K.R. Melo, et al., Development of dextrin-amphotericin B formulations for the treatment of

Leishmaniasis, , https://doi.org/10.1016/j.ijbiomac.2020.03.019 